\title{
Mixing properties of (n-alkanes or esters) + olive oil at different temperatures
}

\author{
C. Gonzalez ${ }^{1}$, J. Lanz ${ }^{1}$, R.S. Andrade ${ }^{2}$, M. Iglesias ${ }^{3, *}$ \\ ${ }^{1}$ Dpto. de Ingeniería Química. Facultad de Farmacia. Universidad del País Vasco, UPV/EHU. Apto. 450. Vitoria. (Spain) \\ ${ }^{2}$ CETENS, Universidade Federal do Recôncavo da Bahia, Feira de Santana, Brasil \\ ${ }^{3}$ Departamento de Engenharia Química, Universidade Federal da Bahia, Salvador, Brasil \\ E-mail: miguel.iglesias@ufba.br
}

Received 5 June 2019, Revised 3 Apr 2020, Accepted 2 May 2020

\begin{abstract}
In the scope of investigating phase equilibria and thermodynamic magnitudes related to equipment design and optimization for edible oil industry, this paper reports refractive index on mixing and ultrasonic velocity of binary systems enclosing a collection of n-alkanes (n-hexane, $n$-heptane, n-octane, n-nonane), or esters (ethyl acetate, vinyl acetate, propyl acetate, isopropyl acetate, butyl acetate) + olive oil at temperatures from 288.15-298.15 K. From these physical properties, the corresponding derived magnitudes, changes of refractive indices on mixing and change of isentropic compressibilities were computed, being fitted by a modified Redlich-Kister polynomial. Due to the fact of industrial processes design to be strongly computer oriented, consideration was also given to quantify how accurate different theoretical estimation models work. The validity of estimation of these thermodynamic properties was tested by different models, which were selected attending to ease of use, wide range of application and accuracy. From the obtained results we can conclude that an adequate agreement between experimental and computed data, both in magnitude or sign in all range of compositions, was observed.
\end{abstract}

\section{Keywords. Refractive index on mixing; isentropic compressibility; $n$-alkane; ester; olive oil; theoretical model}

\section{Introduction}

Knowledge of thermodynamic properties of organic substances with edible oils is of main interest for food industry due to the deep impact of physical properties accuracy on final design and optimal improvement of equipments and processes.

Olive oil is associated to high quality gastronomy and a wide number of scientific publications point out its impact as a healthy daily diet component for heart protection. When extracted, this oil contain a number of impurities which have to be removed to make this suitable for human consumption. Removal of this compounds is done in a series of processes, that includes water degumming, chemical or alkali refining, and then bleaching, deodorization and dewaxing. During processing of the olives to obtain the edible oil, different solvents are used to accelerate dewaxing stage, being a main objective of this stage to minimize the quantity of used solvent, as well as, the necessary contact time [1-2]. Optical and ultrasonic mixing properties provide key physicochemical information of liquid food stuffs allowing insight into the structural organization phenomena. Refractive index is an important optical parameter to analyze the light rays traversing through materials medium and can be used as a tool for determinate the adulteration of oils [3-4]. Aditionally, it is known that changes in the refractive indices of fats and oils has a clear relation to rancid odor development [5]. Ultrasonic investigations also found extensive applications in determining the thermodynamic behavior of liquid mixtures [6-11]. Due to its nondestructive nature, ultrasonic study of liquid mixtures has been extensively carried out, and several researchers correlated the experimental results of ultrasonic velocity and the other thermodynamic parameters derived from it (as isentropic compressibilities) with theoretical models and interpreted the results in terms of molecular interactions between the binary liquid mixture components [12-15].

Common physicochemical properties as density or viscosity are available into open literature for edible oils but data are scarce when we talk about refraction or ultrasonic velocity as a function of composition or in terms of mixing properties as a function of temperature or pressure. Continuing previously research [16-20], in this paper, we present new thermodynamic data of refractive indices on mixing and ultrasonic velocities for organic solvent (n-hexane, n-heptane, n-octane, n-nonane, ethyl acetate, vinyl acetate, propyl acetate, isopropyl acetate, butyl acetate) + olive oil mixtures as a function of temperature (283.15-298.15 K). From these magnitudes, derived properties such as change of refractive indices on mixing and changes of isentropic compressibilities were calculated. The computed derived properties were fitted by a modified Redlich-Kister polynomial. Due to the fact of industrial processes design to be strongly computer oriented, consideration was also given to measure how accurate different theoretical estimation models works. The validity of estimation of these thermodynamic properties were tested by different models (a set of empirical equations for refractive index on mixing and the models of Danusso, Nomoto, Junjie, Impedance Model, Collision Factor and Free Length for ultrasonic velocity estimation), which were selected attending to ease of use, range of application and general accuracy. An adequate 
agreement between experimental and computed data, both in magnitude or sign in all range of compositions, was found.

\section{Experimental}

All the organic solvents used in the preparation of samples were of analytical quality with purity better than 99 mol\% (Merk Lichrosolv). Olive oil was supplied by Koipe (Jaén, Spain), being analyzed by means of a gas chromatograph (Perkin-Elmer model Sigma 3B) equipped with a flame detector. Chromatographic technique and fatty acids chemical procedure analysis were described in previous works [16]. The fatty acids composition obtained was palmitoleic acid, $1.1 \%$; palmitic acid, 16.1 $\%$; stearic acid, $2.4 \%$; oleic acid $73.4 \%$, and linoleic acid, $5.7 \%$; linolenic acid, $1.3 \%$. The uncertainty in mol\% for these results being better than $\pm 0.1 \%$. From this composition, the average molar mass of this oil has been computed using the following expression:

$$
M_{\text {oil }}=3 \cdot\left(\sum_{\mathrm{i}=1}^{\mathrm{NFA}} \mathrm{x}_{\mathrm{i}} \mathrm{M}_{\mathrm{i}}\right)+\mathrm{M}_{\mathrm{CH}-\mathrm{C}-\mathrm{CH}}
$$

where $\mathrm{x}_{\mathrm{i}}$ is the mole fraction and $\mathrm{M}_{\mathrm{i}}$ the molar mass of each fatty acid according to the concentration analysis, NFA the number of fatty acid found by analysis and $\mathrm{M}_{\mathrm{CH}-\mathrm{C}-\mathrm{CH}}$ is the molar mass contributions of the triglyceride molecule axis fraction without three protons. The computed average molar mass in olive oil samples was $870.16 \mathrm{~g} \cdot \mathrm{mol}^{-1}$. The change in molar mass is less than $\pm 1 \mathrm{~g} \cdot \mathrm{mol}^{-1}$. The uncertainties in mole fractions were seen less than \pm 0.0001 in all concentrations. The main physical properties were measured experimentally for each pure component, and the results are shown together with literature values [21-25] in Table 1. GLC tests on the solvents showed solvent purities of higher quality than vendor specifications. The samples were prepared by mass using a Salter ER-182A balance with an accuracy of $510^{-4} \mathrm{~g}$, covering the whole composition ranges of the mixtures. A PolyScience controlled bath model 9510 with a temperature stability of $\pm 10^{-2} \mathrm{~K}$ was used to thermostatize the samples, that stay at the measurement temperature at least one hour before experimental measurements.

The refractive indices were measured by the automatic refractometer ABBEMAT-HP Dr. Kernchen with an uncertainty of \pm 0.00002 . The light source is a light emitting diode (LED) whose beam passes through a polarizing filter, an interference filter $(589.3 \mathrm{~nm})$ and various lenses before it passes through the sapphire prism and encounters the sample. The reflected light (angle critical angle) is led via a lens to the optical sensor, which records the critical angle. The temperature at the boundary region prism-sample is measured by the embedded sensor.

The densities and ultrasonic velocities of pure components and their mixtures were measured with a density and sound analyzer (Anton Paar DSA-48), with an uncertainty of $\pm 0.00001 \mathrm{gcm}^{-3} \mathrm{~ms}^{-1}$ and $\pm 1 \mathrm{~ms}^{-1}$, respectively. The ultrasonic cell determines the ultrasonic velocity of mixtures by means of the sing-around technique. Low intensity ultrasound is used in a very wide range of applications in industry. Many of the applications, such as the measure of density and porosity, involve the measurement of ultrasonic velocities or its attenuation. Lynnworth [26] discusses many of these applications. The principle of operation of the singaround method in this system is that the received pulse triggers another pulse so that a repetitive trigger signal occurs at a rate equal to the reciprocal of the propagation time. The frequency and thus the period between trigger pulses can be very accurately measured and the system is easily automated. However, any timing delays associated with the electronics will show up as errors in the determination of transit time. These electrical delays may be minimized by appropriate signal processing. This system is easily good to $1 \%$ (this is dependent upon path length) for absolute measurements, but is several orders of magnitude more accurate when used as a comparison technique. These procedures are of wide application and have been applied successfully for many types of mixtures. The ultrasonic velocity measuring cell was thermostated with a solid state thermostat using Peltier principle. It consists of a cavity, which is laterally bordered by the receiver and transmitter for the ultrasonic pulses. The surfaces of the ultrasonic transmitter and the receiver are made of stainless steel. A cuvette made of teflon forms all other boundaries of the cavity. The samples are filled and emptied through bores in the cuvette. Therefore all wetted parts consist of teflon and stainless steel only. Apparatus calibration was performed periodically, a double fluid reference being used (Millipore quality degassed water and ambient air) in accordance to technical recommendations.

Additional details of the experimental procedure should be found in earlier published papers, as commented above.

Table 1 Molar mass $\left(\mathrm{gmol}^{-1}\right)$ and physical properties (density $\left(\mathrm{gcm}^{-3}\right)$, refractive index and ultrasonic velocity $\left.\left(\mathrm{ms}^{-1}\right)\right)$ of the pure chemicals at $298.15 \mathrm{~K}$.

\begin{tabular}{|c|c|c|c|c|c|c|c|}
\hline & & \multicolumn{2}{|c|}{ Density } & \multicolumn{2}{|c|}{ Refractive index } & \multicolumn{2}{|c|}{$\begin{array}{c}\text { Ultrasonic } \\
\text { velocity }\end{array}$} \\
\hline & $\begin{array}{c}\text { Molar } \\
\text { mass }\end{array}$ & $\begin{array}{l}\text { This } \\
\text { work }\end{array}$ & Lit. $^{a}$ & $\begin{array}{l}\text { This } \\
\text { work }\end{array}$ & Lit. $^{a}$ & $\begin{array}{c}\text { This } \\
\text { work }\end{array}$ & $\mathrm{Lit}^{b}$ \\
\hline$n$-Hexane & 86.177 & 0.65502 & 0.65484 & 1.37222 & 1.37226 & 1076.9 & 1080 \\
\hline$n$-Heptane & 100.204 & 0.67955 & 0.67946 & 1.38512 & 1.38511 & 1130.3 & 1140 \\
\hline$n$-Octane & 114.231 & 0.6985 & 0.69862 & 1.39513 & 1.39505 & 1172.2 & 1172 \\
\hline$n$ - Nonane & 128.258 & 0.71383 & 0.71375 & 1.40305 & 1.40311 & 1206.3 & 1208 \\
\hline $\begin{array}{l}\text { Ethyl } \\
\text { Acetate }\end{array}$ & 88.106 & 0.8943 & $0.89280^{c}$ & 1.36968 & $1.371^{c}$ & 1139.5 & $1143^{c}$ \\
\hline $\begin{array}{l}\text { Vinyl } \\
\text { Acetate }\end{array}$ & 86.090 & 0.92565 & 0.92634 & 1.39254 & 1.3934 & 1115.7 & - \\
\hline $\begin{array}{l}\text { Propyl } \\
\text { Acetate }\end{array}$ & 102.133 & 0.88206 & $0.88230^{c}$ & 1.38172 & $1.384^{c}$ & 1165.4 & $1149^{c}$ \\
\hline $\begin{array}{l}\text { Isopropyl } \\
\text { Acetate }\end{array}$ & 102.133 & 0.86645 & 0.87020 & 1.37462 & 1.375 & 1103.6 & - \\
\hline $\begin{array}{l}\text { Butyl } \\
\text { Acetate }\end{array}$ & 116.160 & 0.87605 & $0.87560^{c}$ & 1.39184 & $1.393^{c}$ & 1190.5 & $1201^{c}$ \\
\hline Olive oil & 870.16 & 0.90928 & $\begin{array}{l}0.909- \\
0.915^{d}\end{array}$ & 1.46703 & $\begin{array}{c}1.4677- \\
1.4705\end{array}$ & 1448.3 & $1448.2^{e}$ \\
\hline
\end{tabular}

[21], [22], [23], [24], [25]

\section{Results and discussion \\ 3.1 Data correlation}

The experimental data of the change of refractive indices on mixture of the binary systems solvent + olive oil at temperatures from 288.15 to $298.15 \mathrm{~K}$ are presented in Tables 2-3. The measured ultrasonic velocity, the computed isentropic compressibility, and the deviation of this property are shown in Tables 4-5. The deviations of the properties (change of refractive index on mixing and 
change of isentropic compressibility) have been calculated by the following equation:

Table 2. Experimental refractive indices on mixing and change of refractive indices on mixing for n-alkane + olive oil mixtures at 288.15, 293.15 and $298.15 \mathrm{~K}$.

\begin{tabular}{|c|c|c|c|c|c|c|}
\hline \multirow[b]{2}{*}{$\mathrm{x}_{1}$} & \multicolumn{3}{|c|}{$\mathrm{n}_{\mathrm{D}}$} & \multicolumn{3}{|c|}{$\delta \mathrm{n}_{\mathrm{D}}$} \\
\hline & 298.15 & 293.15 & 288.15 & 298.15 & 293.15 & 288.15 \\
\hline \multicolumn{7}{|c|}{ n-Hexane + Olive Oil } \\
\hline 0.0481 & 1.46072 & 1.46283 & 1.46459 & 0.0040 & 0.0042 & 0.0040 \\
\hline 0.1022 & 1.46012 & 1.46204 & 1.46392 & 0.0086 & 0.0087 & 0.0085 \\
\hline 0.1427 & 1.45956 & 1.46144 & 1.46333 & 0.0120 & 0.0120 & 0.0118 \\
\hline 0.1898 & 1.45882 & 1.46081 & 1.46271 & 0.0159 & 0.0159 & 0.0156 \\
\hline 0.2685 & 1.45746 & 1.45945 & 1.46139 & 0.0222 & 0.0221 & 0.0218 \\
\hline 0.3216 & 1.45647 & 1.45835 & 1.46017 & 0.0263 & 0.0262 & 0.0257 \\
\hline 0.3546 & 1.45558 & 1.45745 & 1.45940 & 0.0287 & 0.0284 & 0.0281 \\
\hline 0.3998 & 1.45442 & 1.45633 & 1.45828 & 0.0319 & 0.0317 & 0.0313 \\
\hline 0.4482 & 1.45317 & 1.45483 & 1.45694 & 0.0353 & 0.0349 & 0.0345 \\
\hline 0.5009 & 1.45127 & 1.45312 & 1.45507 & 0.0386 & 0.0382 & 0.0377 \\
\hline 0.5505 & 1.44953 & 1.45137 & 1.45348 & 0.0417 & 0.0413 & 0.0409 \\
\hline 0.6177 & 1.44580 & 1.44787 & 1.44993 & 0.0445 & 0.0443 & 0.0437 \\
\hline 0.6386 & 1.44480 & 1.44690 & 1.44884 & 0.0455 & 0.0453 & 0.0446 \\
\hline 0.6988 & 1.44090 & 1.44300 & 1.44480 & 0.0475 & 0.0472 & 0.0464 \\
\hline 0.7490 & 1.43611 & 1.43840 & 1.44030 & 0.0475 & 0.0475 & 0.0466 \\
\hline 0.7987 & 1.43085 & 1.43308 & 1.43480 & 0.0471 & 0.0469 & 0.0459 \\
\hline 0.8491 & 1.42289 & 1.42481 & 1.42702 & 0.0441 & 0.0435 & 0.0429 \\
\hline 0.8994 & 1.41100 & 1.41305 & 1.41575 & 0.0371 & 0.0366 & 0.0365 \\
\hline 0.9486 & 1.39498 & 1.39683 & 1.39933 & 0.0258 & 0.0252 & 0.0247 \\
\hline \multicolumn{7}{|c|}{ n-Heptane + Olive Oil } \\
\hline 0.0484 & 1.46650 & 1.46840 & 1.47027 & 0.0034 & 0.0034 & 0.0034 \\
\hline 0.0860 & 1.46602 & 1.46787 & 1.46979 & 0.0060 & 0.0059 & 0.0059 \\
\hline 0.1468 & 1.46517 & 1.46704 & 1.46895 & 0.0102 & 0.0100 & 0.0100 \\
\hline 0.1938 & 1.46445 & 1.46638 & 1.46831 & 0.0133 & 0.0132 & 0.0131 \\
\hline 0.2438 & 1.46373 & 1.46551 & 1.46745 & 0.0167 & 0.0164 & 0.0163 \\
\hline 0.3011 & 1.46252 & 1.46440 & 1.46633 & 0.0202 & 0.0199 & 0.0198 \\
\hline 0.3465 & 1.46154 & 1.46347 & 1.46548 & 0.0229 & 0.0227 & 0.0226 \\
\hline 0.4016 & 1.46013 & 1.46211 & 1.46402 & 0.0260 & 0.0258 & 0.0255 \\
\hline 0.4370 & 1.45956 & 1.46110 & 1.46307 & 0.0283 & 0.0277 & 0.0274 \\
\hline 0.5205 & 1.45673 & 1.45846 & 1.46035 & 0.0323 & 0.0318 & 0.0314 \\
\hline 0.5647 & 1.45550 & 1.45667 & 1.45890 & 0.0347 & 0.0336 & 0.0335 \\
\hline 0.6115 & 1.45300 & 1.45445 & 1.45654 & 0.0361 & 0.0352 & 0.0350 \\
\hline 0.6465 & 1.45119 & 1.45258 & 1.45467 & 0.0371 & 0.0362 & 0.0359 \\
\hline 0.7081 & 1.44665 & 1.44855 & 1.45083 & 0.0376 & 0.0371 & 0.0370 \\
\hline 0.7564 & 1.44233 & 1.44465 & 1.44669 & 0.0373 & 0.0372 & 0.0368 \\
\hline 0.7999 & 1.43811 & 1.43981 & 1.44231 & 0.0366 & 0.0359 & 0.0359 \\
\hline 0.8508 & 1.43110 & 1.43307 & 1.43542 & 0.0338 & 0.0333 & 0.0331 \\
\hline 0.9007 & 1.42125 & 1.42345 & 1.42587 & 0.0280 & 0.0277 & 0.0276 \\
\hline 0.9507 & 1.40709 & 1.40973 & 1.41207 & 0.0179 & 0.0180 & 0.0178 \\
\hline \multicolumn{7}{|c|}{ n-Octane + Olive Oil } \\
\hline 0.0499 & 1.46648 & 1.46835 & 1.47025 & 0.0030 & 0.0030 & 0.0030 \\
\hline 0.0957 & 1.46592 & 1.46783 & 1.46966 & 0.0058 & 0.0057 & 0.0056 \\
\hline 0.1481 & 1.46523 & 1.46707 & 1.46899 & 0.0089 & 0.0087 & 0.0087 \\
\hline 0.2025 & 1.46453 & 1.46622 & 1.46816 & 0.0121 & 0.0117 & 0.0117 \\
\hline 0.2467 & 1.46359 & 1.46556 & 1.46740 & 0.0143 & 0.0142 & 0.0141 \\
\hline 0.2923 & 1.46280 & 1.46462 & 1.46656 & 0.0168 & 0.0166 & 0.0164 \\
\hline 0.3437 & 1.46166 & 1.46355 & 1.46556 & 0.0193 & 0.0191 & 0.0191 \\
\hline 0.4016 & 1.46029 & 1.46223 & 1.46415 & 0.0221 & 0.0220 & 0.0218 \\
\hline 0.4855 & 1.45790 & 1.46012 & 1.46207 & 0.0258 & 0.0258 & 0.0256 \\
\hline 0.5056 & 1.45725 & 1.45935 & 1.46112 & 0.0266 & 0.0265 & 0.0261 \\
\hline 0.5498 & 1.45567 & 1.45757 & 1.45955 & 0.0282 & 0.0279 & 0.0276 \\
\hline 0.6116 & 1.45294 & 1.45508 & 1.45692 & 0.0299 & 0.0298 & 0.0294 \\
\hline 0.6438 & 1.45135 & 1.45333 & 1.45533 & 0.0306 & 0.0303 & 0.0301 \\
\hline 0.7021 & 1.44780 & 1.44984 & 1.45183 & 0.0312 & 0.0310 & 0.0307 \\
\hline 0.7484 & 1.44424 & 1.44636 & 1.44841 & 0.0310 & 0.0308 & 0.0305 \\
\hline 0.8034 & 1.43900 & 1.44120 & 1.44328 & 0.0297 & 0.0296 & 0.0293 \\
\hline 0.8533 & 1.43275 & 1.43498 & 1.43711 & 0.0271 & 0.0270 & 0.0267 \\
\hline 0.8971 & 1.42544 & 1.42773 & 1.42993 & 0.0229 & 0.0228 & 0.0226 \\
\hline 0.9471 & 1.41392 & 1.41601 & 1.41866 & 0.0150 & 0.0147 & 0.0148 \\
\hline \multicolumn{7}{|c|}{ n-Nonane + Olive Oil } \\
\hline 0.0454 & 1.46656 & 1.46837 & 1.47037 & 0.0024 & 0.0023 & 0.0024 \\
\hline 0.1026 & 1.46589 & 1.46774 & 1.46961 & 0.0054 & 0.0053 & 0.0053 \\
\hline 0.1405 & 1.46530 & 1.46717 & 1.46909 & 0.0073 & 0.0072 & 0.0071 \\
\hline 0.1966 & 1.46450 & 1.46633 & 1.46828 & 0.0100 & 0.0099 & 0.0099 \\
\hline 0.2527 & 1.46357 & 1.46546 & 1.46731 & 0.0127 & 0.0126 & 0.0124 \\
\hline 0.3100 & 1.46246 & 1.46438 & 1.46627 & 0.0153 & 0.0151 & 0.0150 \\
\hline 0.3616 & 1.46133 & 1.46327 & 1.46523 & 0.0174 & 0.0173 & 0.0172 \\
\hline 0.4165 & 1.45998 & 1.46193 & 1.46388 & 0.0196 & 0.0195 & 0.0193 \\
\hline 0.4360 & 1.45961 & 1.46145 & 1.46341 & 0.0205 & 0.0202 & 0.0201 \\
\hline
\end{tabular}

\begin{tabular}{llll|lll}
0.5161 & 1.45709 & 1.45907 & 1.46100 & 0.0231 & 0.0229 & 0.0227 \\
0.5502 & 1.45586 & 1.45785 & 1.45983 & 0.0240 & 0.0239 & 0.0237 \\
0.6060 & 1.45358 & 1.45560 & 1.45755 & 0.0253 & 0.0252 & 0.0249 \\
0.6440 & 1.45180 & 1.45372 & 1.45579 & 0.0260 & 0.0257 & 0.0255 \\
0.6909 & 1.44920 & 1.45129 & 1.45317 & 0.0264 & 0.0262 & 0.0259 \\
0.7438 & 1.44564 & 1.44765 & 1.44971 & 0.0262 & 0.0260 & 0.0257 \\
0.7955 & 1.44117 & 1.44326 & 1.44541 & 0.0250 & 0.0249 & 0.0247 \\
0.8521 & 1.43492 & 1.43713 & 1.43926 & 0.0224 & 0.0223 & 0.0221 \\
0.8940 & 1.42870 & 1.43110 & 1.43328 & 0.0189 & 0.0190 & 0.0188 \\
0.9531 & 1.41700 & 1.41911 & 1.42121 & 0.0110 & 0.0107 & 0.0104 \\
\hline
\end{tabular}

$\delta \mathrm{P}=\mathrm{P}_{\text {mix }}-\sum_{\mathrm{i}=1}^{\mathrm{N}} \mathrm{x}_{\mathrm{i}} \mathrm{P}_{\mathrm{i}}$

In this equation, $\delta \mathrm{P}$ means the deviation of a magnitude $\mathrm{P}$ (refractive index, $\mathrm{n}_{\mathrm{D}}$, or isentropic compressibility, $\kappa_{\mathrm{S}}$, computed by the Newton-Laplace equation), $\mathrm{P}_{\mathrm{i}}$ is the pure solvent magnitude, $\mathrm{P}_{\operatorname{mix}}$ is the value of a property for a mixture, $\mathrm{x}_{\mathrm{i}}$ is the mole fraction, and $\mathrm{N}$ is the number of components into the mixture.

In Figures 1-2, the derived properties of the studied systems have been plotted along the mole fraction at $298.15 \mathrm{~K}$. The derived magnitudes of the binary mixtures were fitted using a authors' proposed modification of the Redlich-Kister expression:

$\delta \mathrm{P}_{\mathrm{ij}}=\mathrm{x}_{\mathrm{i}} \mathrm{x}_{\mathrm{j}} \cdot\left(\sum_{\mathrm{p}=0}^{\mathrm{S}}\left(\sum_{\mathrm{j}=0}^{\mathrm{M}} \mathrm{B}_{\mathrm{pj}} \mathrm{T}^{\mathrm{j}}\right) \cdot\left(\mathrm{x}_{\mathrm{i}}-\mathrm{x}_{\mathrm{j}}\right)^{\mathrm{p}}\right)$

where $\delta \mathrm{P}_{\mathrm{ij}}$ is the derived property (change of refractive index on mixing or change of isentropic compressibility), $\mathrm{B}_{\mathrm{pj}}$ are the fitting parameters obtained by the unweighted least squared method applying a fitting Marquardt algorithm (all the points weighting the same), and $\mathrm{S}$ is the degree of the polynomial expansion.

The root mean square deviations were computed using eq. 4 , where $\mathrm{z}$ is the value of the property, and $\mathrm{n}_{\mathrm{DAT}}$ is the number of experimental data of each experimental collection. In both studied properties, the applied fitting equation fits accurately to the experimental data, as could be observed in Figure 1.

The fitting parameters are gathered into Table 6 , with the corresponding root mean square deviations $(\sigma)$ expressed by eq. 4 .

$\sigma=\left(\frac{\sum_{\mathrm{i}=1}^{\mathrm{n}_{\mathrm{DAT}}}\left(\mathrm{z}_{\mathrm{exp}}-\mathrm{z}_{\mathrm{pred}}\right)^{2}}{\mathrm{n}_{\mathrm{DAT}}}\right)^{1 / 2}$

The thermodynamic properties have been measured at three temperatures, 288.15, 293.15 and $298.15 \mathrm{~K}$. In Figure 1, the change of refractive indices on mixing with (n-alkane or ester solvent) mole fraction at $298.15 \mathrm{~K}$, should be observed. Figure 2 shows the change of isentropic compressibilities with (n-alkane or ester) mole fraction at $298.15 \mathrm{~K}$.

Figure 3 gathers the composition/temperature trend for nhexane and ethyl acetate for both derived properties. 
Table 3. Experimental refractive indices and change of refractive index of mixing for esters + olive oil mixtures at 288.15, 293.15 and $298.15 \mathrm{~K}$.

\begin{tabular}{lllllll}
\hline & \multicolumn{3}{c}{$\mathrm{n}_{\mathrm{D}}$} & \multicolumn{3}{c}{$\delta \mathrm{n}_{\mathrm{D}}$} \\
\cline { 2 - 6 } & 298.15 & 293.15 & 288.15 & 298.15 & 293.15 & 288.15
\end{tabular}

\begin{tabular}{llll|lll}
\hline \multicolumn{7}{c}{ Ethyl Acetate + Olive Oil } \\
\hline 0.0603 & 1.46636 & 1.46828 & 1.46965 & 0.0052 & 0.0052 & 0.0046 \\
0.1185 & 1.46572 & 1.46762 & 1.46935 & 0.0102 & 0.0101 & 0.0099 \\
0.1541 & 1.46530 & 1.46711 & 1.46891 & 0.0133 & 0.0131 & 0.0129 \\
0.2014 & 1.46455 & 1.46640 & 1.46818 & 0.0171 & 0.0169 & 0.0167 \\
0.2478 & 1.46405 & 1.46571 & 1.46743 & 0.0211 & 0.0207 & 0.0204 \\
0.3094 & 1.46269 & 1.46460 & 1.46655 & 0.0258 & 0.0255 & 0.0254 \\
0.3497 & 1.46187 & 1.46380 & 1.46568 & 0.0289 & 0.0286 & 0.0284 \\
0.3962 & 1.46100 & 1.46273 & 1.46460 & 0.0325 & 0.0321 & 0.0318 \\
0.4470 & 1.45960 & 1.46148 & 1.46324 & 0.0361 & 0.0357 & 0.0353 \\
0.4982 & 1.45794 & 1.45983 & 1.46169 & 0.0394 & 0.0390 & 0.0386 \\
0.5326 & 1.45672 & 1.45867 & 1.46049 & 0.0415 & 0.0412 & 0.0407 \\
0.6229 & 1.45270 & 1.45473 & 1.45671 & 0.0463 & 0.0459 & 0.0456 \\
0.6498 & 1.45128 & 1.45319 & 1.45512 & 0.0475 & 0.0470 & 0.0466 \\
0.7133 & 1.44700 & 1.44896 & 1.45087 & 0.0494 & 0.0489 & 0.0484 \\
0.7511 & 1.44370 & 1.44580 & 1.44780 & 0.0498 & 0.0494 & 0.0490 \\
0.8039 & 1.43788 & 1.43974 & 1.44174 & 0.0491 & 0.0484 & 0.0480 \\
0.8506 & 1.43056 & 1.43277 & 1.43487 & 0.0463 & 0.0460 & 0.0456 \\
0.8992 & 1.42005 & 1.42215 & 1.42425 & 0.0406 & 0.0400 & 0.0396 \\
0.9517 & 1.40140 & 1.40372 & 1.40597 & 0.0270 & 0.0267 & 0.0264 \\
\hline \multicolumn{7}{c}{ Vinyl Acetate + Olive Oil } \\
\hline 0.0688 & 1.46652 & 1.46840 & 1.47029 & 0.0046 & 0.0045 & 0.0045 \\
\hline
\end{tabular}

\begin{tabular}{llll|lll}
\hline 0.0688 & 1.46652 & 1.46840 & 1.47029 & 0.0046 & 0.0045 & 0.0045 \\
0.1187 & 1.46611 & 1.46796 & 1.46988 & 0.0079 & 0.0078 & 0.0077
\end{tabular}

\begin{tabular}{llll|lll}
0.1608 & 1.46567 & 1.46756 & 1.46944 & 0.0106 & 0.0105 & 0.0103
\end{tabular}

\begin{tabular}{llll|lll}
0.1854 & 1.46545 & 1.46728 & 1.46920 & 0.0122 & 0.0120 & 0.0119
\end{tabular}

\begin{tabular}{llll|lll}
0.2494 & 1.46467 & 1.46653 & 1.46846 & 0.0162 & 0.0159 & 0.0158
\end{tabular}

\begin{tabular}{llll|lll}
0.3000 & 1.46398 & 1.46593 & 1.46790 & 0.0193 & 0.0191 & 0.0189
\end{tabular}

\begin{tabular}{llll|llll}
0.3468 & 1.46335 & 1.46526 & 1.46714 & 0.0222 & 0.0218 & 0.0215
\end{tabular}

\begin{tabular}{llll|lll}
0.3816 & 1.46275 & 1.46468 & 1.46659 & 0.0241 & 0.0238 & 0.0235
\end{tabular}

$\begin{array}{llllllll}0.4391 & 1.46170 & 1.46357 & 1.46557 & 0.0274 & 0.0269 & 0.0266\end{array}$

\begin{tabular}{llll|lll}
0.4951 & 1.46047 & 1.46236 & 1.46435 & 0.0303 & 0.0298 & 0.0295
\end{tabular}

\begin{tabular}{llll|lll}
0.5627 & 1.45859 & 1.46046 & 1.46253 & 0.0335 & 0.0329 & 0.0326
\end{tabular}

\begin{tabular}{llll|lll}
0.6125 & 1.45692 & 1.45895 & 1.46087 & 0.0355 & 0.0350 & 0.0345
\end{tabular}

\begin{tabular}{llll|lll}
0.6558 & 1.45530 & 1.45711 & 1.45922 & 0.0371 & 0.0364 & 0.0360
\end{tabular}

$\begin{array}{llllllll}0.6924 & 1.45339 & 1.45540 & 1.45751 & 0.0379 & 0.0374 & 0.0369\end{array}$

\begin{tabular}{llll|lll}
0.7616 & 1.44903 & 1.45122 & 1.45316 & 0.0387 & 0.0383 & 0.0376
\end{tabular}

\begin{tabular}{llll|lll}
0.8063 & 1.44508 & 1.44718 & 1.44940 & 0.0381 & 0.0375 & 0.0371
\end{tabular}

\begin{tabular}{llll|lll}
0.8542 & 1.43937 & 1.44169 & 1.44391 & 0.0360 & 0.0355 & 0.0351
\end{tabular}

\begin{tabular}{llll|lll}
0.9024 & 1.43101 & 1.43334 & 1.43577 & 0.0312 & 0.0307 & 0.0304
\end{tabular}

\begin{tabular}{llll|lll}
0.9491 & 1.41828 & 1.42087 & 1.42340 & 0.0219 & 0.0217 & 0.0215 \\
\hline
\end{tabular}

\begin{tabular}{llll|lll}
\hline \multicolumn{7}{c}{ Propyl Acetate + Olive Oil } \\
\hline 0.0490 & 1.46650 & 1.46842 & 1.47029 & 0.0037 & 0.0036 & 0.0036 \\
0.1143 & 1.46579 & 1.46769 & 1.46942 & 0.0085 & 0.0084 & 0.0082 \\
0.1644 & 1.46510 & 1.46701 & 1.46885 & 0.0121 & 0.0120 & 0.0119 \\
0.1946 & 1.46468 & 1.46657 & 1.46852 & 0.0143 & 0.0141 & 0.0141 \\
0.2645 & 1.46349 & 1.46543 & 1.46727 & 0.0190 & 0.0189 & 0.0187 \\
0.3010 & 1.46282 & 1.46468 & 1.46688 & 0.0215 & 0.0212 & 0.0214 \\
0.3542 & 1.46175 & 1.46361 & 1.46567 & 0.0249 & 0.0247 & 0.0246 \\
0.4183 & 1.46024 & 1.46214 & 1.46407 & 0.0289 & 0.0286 & 0.0284 \\
0.4364 & 1.45973 & 1.46170 & 1.46362 & 0.0299 & 0.0297 & 0.0295 \\
0.4897 & 1.45834 & 1.46010 & 1.46206 & 0.0331 & 0.0326 & 0.0324 \\
0.5301 & 1.45680 & 1.45887 & 1.46069 & 0.0350 & 0.0348 & 0.0344 \\
0.5904 & 1.45428 & 1.45640 & 1.45831 & 0.0376 & 0.0375 & 0.0371 \\
0.6386 & 1.45198 & 1.45401 & 1.45599 & 0.0394 & 0.0392 & 0.0388 \\
0.7207 & 1.44691 & 1.44879 & 1.45106 & 0.0414 & 0.0409 & 0.0408 \\
0.7577 & 1.44355 & 1.44573 & 1.44771 & 0.0412 & 0.0410 & 0.0406 \\
0.8054 & 1.43855 & 1.44063 & 1.44282 & 0.0402 & 0.0399 & 0.0397 \\
0.8614 & 1.43026 & 1.43240 & 1.43467 & 0.0367 & 0.0364 & 0.0362 \\
0.9013 & 1.42211 & 1.42440 & 1.42669 & 0.0320 & 0.0318 & 0.0316 \\
0.9551 & 1.40544 & 1.40789 & 1.41021 & 0.0199 & 0.0199 & 0.0196 \\
\hline
\end{tabular}

\begin{tabular}{llll|l}
0.9551 & 1.40544 & 1.40789 & 1.41021 & 0.0199 \\
\hline & & Isopropyl Acetate + Olive Oil
\end{tabular}

\begin{tabular}{llll|lll}
\hline 0.0543 & 1.46640 & 1.46831 & 1.47020 & 0.0044 & 0.0044 & 0.0043 \\
0.1005 & 1.46583 & 1.46772 & 1.46960 & 0.0081 & 0.0080 & 0.0079 \\
0.1426 & 1.46542 & 1.46711 & 1.46913 & 0.0116 & 0.0112 & 0.0113 \\
0.2007 & 1.46430 & 1.46631 & 1.46816 & 0.0158 & 0.0158 & 0.0156 \\
0.2342 & 1.46389 & 1.46569 & 1.46757 & 0.0185 & 0.0182 & 0.0181 \\
0.2985 & 1.46251 & 1.46446 & 1.46645 & 0.0231 & 0.0229 & 0.0228 \\
0.3537 & 1.46137 & 1.46355 & 1.46514 & 0.0270 & 0.0270 & 0.0265 \\
0.4169 & 1.45963 & 1.46183 & 1.46372 & 0.0311 & 0.0311 & 0.0308 \\
0.4440 & 1.45894 & 1.46082 & 1.46278 & 0.0329 & 0.0326 & 0.0323 \\
0.5332 & 1.45581 & 1.45776 & 1.45984 & 0.0380 & 0.0377 & 0.0375 \\
0.5669 & 1.45432 & 1.45637 & 1.45831 & 0.0397 & 0.0394 & 0.0390 \\
0.6031 & 1.45274 & 1.45489 & 1.45664 & 0.0414 & 0.0412 & 0.0407
\end{tabular}

\begin{tabular}{llll|lll}
0.6462 & 1.45035 & 1.45227 & 1.45422 & 0.0430 & 0.0426 & 0.0422 \\
0.7113 & 1.44602 & 1.44791 & 1.45007 & 0.0447 & 0.0442 & 0.0439 \\
0.7477 & 1.44255 & 1.44456 & 1.44675 & 0.0446 & 0.0441 & 0.0439 \\
0.8052 & 1.43604 & 1.43835 & 1.44034 & 0.0434 & 0.0432 & 0.0427 \\
0.8484 & 1.42965 & 1.43184 & 1.43402 & 0.0410 & 0.0407 & 0.0403 \\
0.9088 & 1.41620 & 1.41856 & 1.42080 & 0.0332 & 0.0329 & 0.0326 \\
0.9530 & 1.40121 & 1.40360 & 1.40617 & 0.0222 & 0.0220 & 0.0220 \\
\hline \multicolumn{7}{c}{ Butyl Acetate + Olive Oil } \\
\hline 0.0585 & 1.46639 & 1.46831 & 1.47019 & 0.0038 & 0.0037 & 0.0037 \\
0.0856 & 1.46596 & 1.46799 & 1.46987 & 0.0054 & 0.0054 & 0.0054 \\
0.1379 & 1.46544 & 1.46728 & 1.46924 & 0.0088 & 0.0086 & 0.0087 \\
0.1920 & 1.46464 & 1.46655 & 1.46846 & 0.0120 & 0.0119 & 0.0119 \\
0.2415 & 1.46390 & 1.46579 & 1.46771 & 0.0150 & 0.0149 & 0.0149 \\
0.2877 & 1.46310 & 1.46495 & 1.46688 & 0.0177 & 0.0175 & 0.0175 \\
0.3426 & 1.46202 & 1.46387 & 1.46587 & 0.0207 & 0.0205 & 0.0205 \\
0.3938 & 1.46081 & 1.46283 & 1.46472 & 0.0234 & 0.0233 & 0.0232 \\
0.4523 & 1.45937 & 1.46124 & 1.46325 & 0.0263 & 0.0261 & 0.0260 \\
0.5061 & 1.45776 & 1.45969 & 1.46166 & 0.0288 & 0.0285 & 0.0285 \\
0.5493 & 1.45625 & 1.45817 & 1.46012 & 0.0305 & 0.0302 & 0.0301 \\
0.6015 & 1.45414 & 1.45608 & 1.45804 & 0.0323 & 0.0320 & 0.0319 \\
0.6479 & 1.45185 & 1.45381 & 1.45584 & 0.0335 & 0.0332 & 0.0332 \\
0.6921 & 1.44927 & 1.45131 & 1.45331 & 0.0343 & 0.0340 & 0.0339 \\
0.7552 & 1.44467 & 1.44666 & 1.44872 & 0.0344 & 0.0341 & 0.0340 \\
0.7929 & 1.44105 & 1.44313 & 1.44524 & 0.0336 & 0.0334 & 0.0333 \\
0.8474 & 1.43444 & 1.43656 & 1.43866 & 0.0311 & 0.0309 & 0.0308 \\
0.9080 & 1.42362 & 1.42581 & 1.42799 & 0.0249 & 0.0246 & 0.0246 \\
0.9507 & 1.41230 & 1.41459 & 1.41683 & 0.0168 & 0.0166 & 0.0166 \\
\hline
\end{tabular}

\subsection{Physical properties estimation}

Due to the strong dependence of design and optimization of chemical processes on computer calculations, the availability of accurate and tested methods is of increasing relevance. In what is referred to estimate the refractive indices on mixing, different rules were applied, which are dependent on the pure values at the studied temperature. The experimental refractive indices on mixing have been compared with those estimated by means of the mixing rules proposed by Lorentz-Lorenz (eq. 5), Dale-Gladstone (eq. 6), Eykman (eq. 7), Arago-Biot (eq. 8) Newton (eq. 9), Oster (eq. 10), Eyring-John (eq. 11), Weiner (eq. 12) and Heller (eq. 13) [27]:

$$
\frac{\mathrm{n}_{\mathrm{D}}^{2}-1}{\mathrm{n}_{\mathrm{D}}^{2}+2}=\sum_{\mathrm{i}=1}^{\mathrm{N}}\left[\phi_{\mathrm{i}}\left(\frac{\mathrm{n}_{\mathrm{Di}}^{2}-1}{\mathrm{n}_{\mathrm{Di}}^{2}+2}\right)\right]
$$

$$
\frac{\mathrm{n}_{\mathrm{D}}^{2}-1}{\mathrm{n}_{\mathrm{D}}^{2}+2}=\sum_{\mathrm{i}=1}^{\mathrm{N}}\left[\phi_{\mathrm{i}}\left(\frac{\mathrm{n}_{\mathrm{Di}}^{2}-1}{\mathrm{n}_{\mathrm{Di}}^{2}+2}\right)\right]
$$

$$
\mathrm{n}_{\mathrm{D}}-1=\sum_{\mathrm{i}=1}^{\mathrm{N}}\left[\phi_{\mathrm{i}}\left(\mathrm{n}_{\mathrm{Di}}-1\right)\right]
$$

$$
\frac{\mathrm{n}_{\mathrm{D}}^{2}-1}{\mathrm{n}_{\mathrm{D}}^{2}+0.4}=\sum_{\mathrm{i}=1}^{\mathrm{N}}\left[\phi_{\mathrm{i}}\left(\frac{\mathrm{n}_{\mathrm{Di}}^{2}-1}{\mathrm{n}_{\mathrm{Di}}^{2}+0.4}\right)\right]
$$

$$
\mathrm{n}_{\mathrm{D}}=\sum_{\mathrm{i}=1}^{\mathrm{N}}\left(\phi_{\mathrm{i}} \mathrm{n}_{\mathrm{Di}}\right)
$$

$$
\mathrm{n}_{\mathrm{D}}^{2}-1=\sum_{\mathrm{i}=1}^{\mathrm{N}}\left[\phi_{\mathrm{i}}\left(\mathrm{n}_{\mathrm{Di}}^{2}-1\right)\right]
$$


$\frac{\left(\mathrm{n}_{\mathrm{D}}^{2}-1\right)\left(2 \mathrm{n}_{\mathrm{D}}^{2}+1\right)}{\mathrm{n}_{\mathrm{D}}^{2}}=\sum_{\mathrm{i}=1}^{\mathrm{N}}\left[\phi_{\mathrm{i}} \frac{\left(\mathrm{n}_{\mathrm{Di}}^{2}-1\right)\left(2 \mathrm{n}_{\mathrm{Di}}^{2}+1\right)}{\mathrm{n}_{\mathrm{Di}}^{2}}\right]$

and for binary mixtures

$$
\begin{aligned}
& \mathrm{n}_{\mathrm{D}}=\mathrm{n}_{1} \phi_{1}^{2}+2\left(\mathrm{n}_{1} \mathrm{n}_{2}\right)^{1 / 2} \phi_{1} \phi_{1}+\mathrm{n}_{2} \phi_{2}^{2} \\
& \frac{\mathrm{n}_{\mathrm{D}}^{2}-\mathrm{n}_{\mathrm{D} 1}^{2}}{\mathrm{n}_{\mathrm{D}}^{2}+2 \mathrm{n}_{\mathrm{D} 1}^{2}}=\left[\phi_{2} \frac{\mathrm{n}_{\mathrm{D} 2}^{2}-\mathrm{n}_{\mathrm{D} 1}^{2}}{\mathrm{n}_{\mathrm{D} 2}^{2}+2 \mathrm{n}_{\mathrm{D} 1}^{2}}\right]
\end{aligned}
$$

The results of the comparison with experimental data at 298.15 K appear in the Table 7, in which the root mean square deviations (eq. 4) between experimental and estimated data are shown. A good agreement is observed, appearing the lower deviations for Arago-Biot, Gladstone-Dale, Newton and Oster equations for all studied solvents.

Table 4 Experimental data of ultrasonic velocity, isentropic compressibilities and change of isentropic compressibilities for $n$ -

\begin{tabular}{|c|c|c|c|c|c|c|c|c|c|}
\hline \multicolumn{4}{|c|}{$\mathrm{u} / \mathrm{m} \cdot \mathrm{s}^{-1}$} & \multicolumn{3}{|c|}{$\kappa_{\delta} / \mathrm{TPa}^{-1}$} & \multicolumn{3}{|c|}{$\delta \kappa_{\mathrm{s}} / \mathrm{TPa}^{-1}$} \\
\hline $\mathrm{X}_{1}$ & 298.15 & 293.15 & 288.15 & 298.15 & 293.15 & 288.15 & 298.15 & 293.15 & 288.15 \\
\hline \multicolumn{10}{|c|}{ n-Hexane + Olive oil } \\
\hline 0.0470 & 1445.4 & 1462.5 & 1479.3 & 527.7 & 513.3 & \begin{tabular}{|c|}
499.9 \\
\end{tabular} & -34.3 & -32.2 & $\begin{array}{l}-30.0 \\
\end{array}$ \\
\hline 0.1109 & 1441.3 & 1458.2 & 1475.4 & 531.9 & 517.7 & 503.8 & -80.6 & -75.4 & -70.7 \\
\hline 0.2048 & 1434.3 & 1451.4 & 1468.4 & 539.5 & 524.9 & 510.8 & -147.5 & -138.0 & -129.4 \\
\hline 0.3153 & 1423.9 & 1441.3 & 1458.1 & 551.1 & 535.8 & 521.5 & -223.5 & -209.5 & -196.0 \\
\hline 0.4098 & 1412.9 & 1430.1 & 1447.3 & 563.7 & 548.1 & 533.1 & -285.9 & -267.5 & -250.6 \\
\hline 0.4527 & 1406.8 & 1424.1 & 1441.2 & 570.9 & 554.9 & 539.7 & -312.6 & -292.6 & -273.9 \\
\hline 0.4998 & 1399.3 & 1416.6 & 1433.8 & 579.9 & 563.6 & 548.0 & -341.0 & -319.0 & -298.6 \\
\hline 0.5616 & 1388.3 & 1405.6 & 1423.0 & 593.4 & 576.6 & 560.4 & -376.4 & -352.0 & -329.4 \\
\hline 0.5972 & 1380.1 & 1397.6 & 1414.9 & 603.8 & 586.4 & 569.9 & -394.3 & -368.7 & -344.8 \\
\hline 0.7010 & 1348.7 & 1366.6 & 1384.2 & 646.2 & 626.7 & 608.4 & -434.1 & -405.7 & -379.0 \\
\hline 0.7970 & 1306.7 & 1325.9 & 1343.9 & 709.6 & 685.8 & 664.7 & -446.9 & -418.0 & -389.8 \\
\hline 0.9027 & 1227.3 & 1246.7 & 1265.0 & 857.6 & 827.0 & 799.2 & -382.6 & -355.5 & -329.2 \\
\hline 0.9493 & 1153.4 & 1174.4 & 1195.1 & 1042.9 & 1000.2 & 960.3 & -234.3 & -217.0 & -200.7 \\
\hline \multicolumn{10}{|c|}{ n-Heptane + Olive Oil } \\
\hline 0.0448 & 1445.6 & 1462.8 & 1479.4 & 527.5 & 513.0 & 499.7 & -25.2 & -23.9 & -22.3 \\
\hline 0.0964 & 1442.4 & 1459.6 & 1476.2 & 530.9 & 516.4 & 502.9 & -54.2 & -51.1 & -48.0 \\
\hline 0.1904 & 1435.8 & 1453.0 & 1469.7 & 538.1 & 523.4 & 509.6 & -105.9 & -99.6 & -94.0 \\
\hline 0.3087 & 1425.5 & 1442.7 & 1459.5 & 549.8 & 534.7 & 520.5 & -168.4 & -158.2 & -149.4 \\
\hline 0.4090 & 1414.4 & 1431.6 & 1448.5 & 562.7 & 547.3 & 532.4 & -218.3 & -204.9 & -193.7 \\
\hline 0.4577 & 1407.9 & 1425.1 & 1442.1 & 570.6 & 554.8 & 539.6 & -241.1 & -226.2 & -213.8 \\
\hline 0.4968 & 1402.0 & 1419.2 & 1436.3 & 577.8 & 561.7 & 546.2 & -258.3 & -242.4 & -229.0 \\
\hline 0.5463 & 1393.2 & 1410.4 & 1427.6 & 588.8 & 572.4 & 556.4 & -278.4 & -261.0 & -246.6 \\
\hline 0.6175 & 1378.7 & 1396.1 & 1413.2 & 607.6 & 590.2 & 573.7 & -304.2 & -285.2 & -269.2 \\
\hline 0.7016 & 1356.3 & 1374.2 & 1391.5 & 638.3 & 619.3 & 601.4 & -326.2 & -305.8 & -288.6 \\
\hline 0.7922 & 1320.6 & 1337.3 & 1356.1 & 692.3 & 673.0 & 651.1 & -329.0 & -305.7 & -289.6 \\
\hline 0.8903 & 1258.6 & 1277.6 & 1296.5 & 803.7 & 776.3 & 750.1 & -279.2 & -260.3 & -245.6 \\
\hline 0.9499 & 1199.6 & 1220.1 & 1240.2 & 937.7 & 901.9 & 868.0 & -182.6 & -170.0 & -161.1 \\
\hline \multicolumn{10}{|c|}{ n-Octane + Olive Oil } \\
\hline 0.0408 & 1445.8 & 1463.8 & 1478.1 & 527.1 & 512.2 & 501.3 & -18.7 & -18.3 & -14.7 \\
\hline 0.1123 & 1441.7 & 1458.8 & 1475.5 & 531.8 & 517.4 & 503.9 & -51.0 & -48.1 & -45.2 \\
\hline 0.1931 & 1436.1 & 1453.3 & 1469.9 & 538.1 & 523.5 & 509.7 & -86.6 & -81.6 & -76.9 \\
\hline 0.3038 & 1426.9 & 1444.1 & 1460.9 & 548.7 & 533.7 & 519.5 & -133.3 & -125.6 & -118.6 \\
\hline 0.4047 & 1416.3 & 1433.5 & 1450.4 & 561.3 & 545.9 & 531.1 & -172.9 & -162.8 & -153.7 \\
\hline 0.4554 & 1409.9 & 1427.1 & 1444.1 & 569.1 & 553.4 & 538.3 & -191.4 & -180.1 & -170.1 \\
\hline 0.5088 & 1402.3 & 1419.4 & 1436.5 & 578.7 & 562.7 & 547.1 & -209.6 & -197.0 & -186.0 \\
\hline 0.5474 & 1396.1 & 1413.3 & 1430.4 & 586.6 & 570.3 & 554.5 & -221.6 & -208.3 & -196.6 \\
\hline 0.6000 & 1386.3 & 1403.2 & 1420.8 & 599.5 & 583.0 & 566.3 & -236.0 & -221.3 & -209.2 \\
\hline 0.7121 & 1358.4 & 1376.0 & 1393.5 & 638.4 & 619.7 & 601.7 & -255.1 & -239.6 & -225.9 \\
\hline 0.8047 & 1324.4 & 1341.8 & 1360.1 & 691.1 & 670.9 & 649.7 & -250.3 & -233.7 & -220.8 \\
\hline 0.9090 & 1263.4 & 1282.4 & 1300.4 & 804.3 & 777.0 & 752.0 & -191.2 & -178.7 & -167.0 \\
\hline 0.9501 & 1228.3 & 1247.2 & 1266.7 & 883.8 & 853.6 & 822.6 & -133.0 & -122.1 & -115.4 \\
\hline \multicolumn{10}{|c|}{ n-Nonane + Olive Oil } \\
\hline 0.0435 & 1446.0 & 1464.3 & 1479.7 & 527.1 & 511.6 & 499.5 & -16.6 & -17.0 & -14.6 \\
\hline 0.1183 & 1441.6 & 1458.4 & 1475.5 & 532.1 & 517.9 & 504.0 & -44.4 & -41.8 & -39.6 \\
\hline 0.1993 & 1436.3 & 1453.1 & 1470.1 & 538.2 & 523.9 & 509.9 & -73.7 & -69.5 & -65.7 \\
\hline 0.3006 & 1428.1 & 1444.9 & 1462.1 & 547.8 & 533.2 & 518.7 & -108.4 & -102.4 & -96.9 \\
\hline 0.4048 & 1417.9 & 1434.7 & 1452.0 & 560.3 & 545.2 & 530.1 & -141.6 & -133.6 & -126.5 \\
\hline 0.4571 & 1410.1 & 1428.2 & 1445.9 & 569.5 & 553.0 & 537.3 & -155.3 & -147.6 & -140.0 \\
\hline 0.5037 & 1405.2 & 1422.4 & 1439.7 & 576.2 & 560.2 & 544.6 & -169.0 & -159.8 & -151.1 \\
\hline 0.5525 & 1397.8 & 1415.0 & 1432.4 & 585.9 & 569.5 & 553.5 & -180.7 & -170.8 & -161.4 \\
\hline 0.6059 & 1388.4 & 1405.6 & 1423.1 & 598.5 & 581.6 & 565.1 & -191.5 & -180.8 & -170.9 \\
\hline 0.7038 & 1366.2 & 1383.8 & 1401.3 & 629.9 & 611.5 & 593.8 & -203.0 & -191.7 & -180.7 \\
\hline 0.8017 & 1335.0 & 1352.9 & 1371.0 & 678.4 & 657.8 & 637.8 & -197.3 & -186.1 & -175.4 \\
\hline 0.9037 & 1284.3 & 1303.3 & 1322.1 & 770.8 & 745.0 & 720.6 & -149.5 & -141.2 & -132.8 \\
\hline 0.9528 & 1249.9 & 1269.2 & 1288.7 & 846.6 & 817.0 & 788.6 & -95.3 & -89.6 & -84.2 \\
\hline
\end{tabular}
alkane + olive oil mixtures at 288.15,293.15 and $298.15 \mathrm{~K}$.
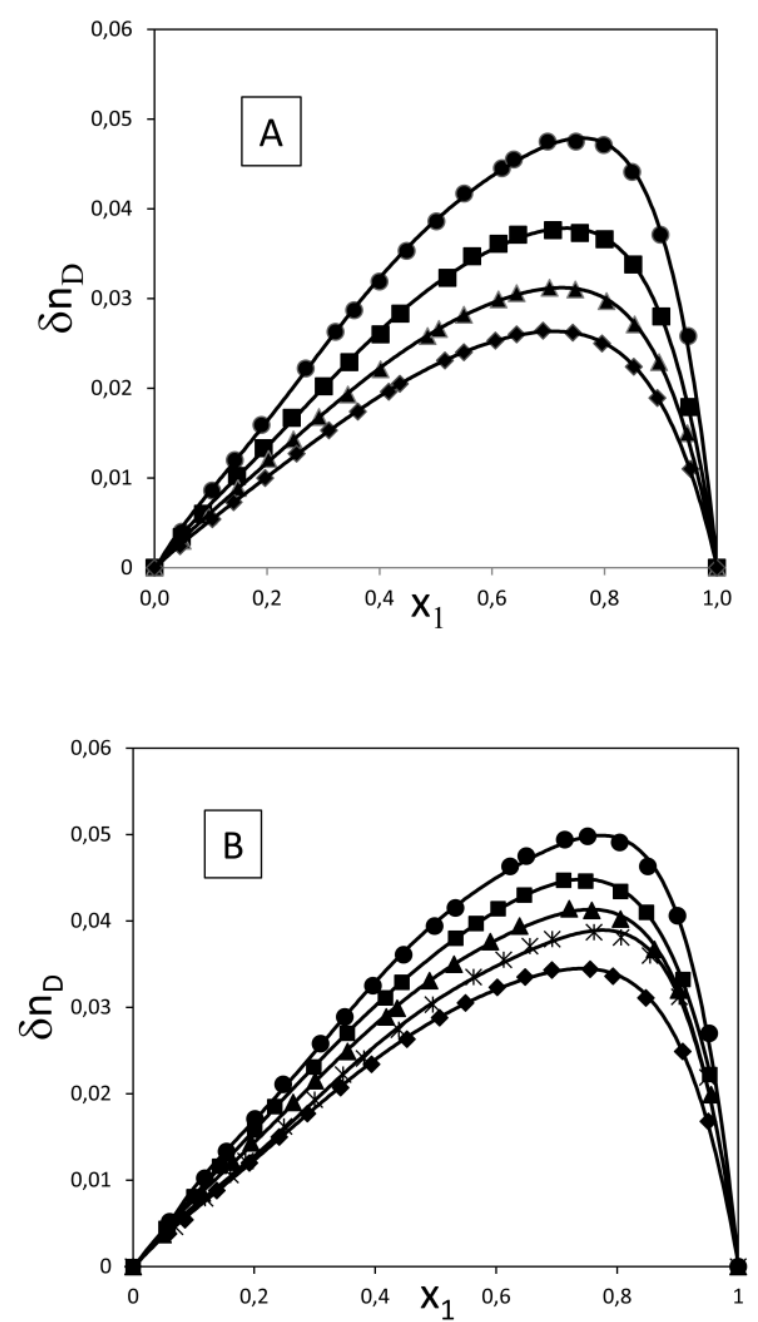

Figure 1. Change of refractive index on mixing versus

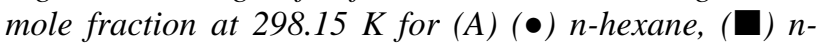
heptane, ( $\mathbf{\Delta})$ n-octane, $(\bullet)$ n-nonane) (1)+olive oil (2) and (一, Eq. 3) fitting curves and for $(B)((\bullet)$ ethyl acetate, $(*)$ vinyl actetate, $(\mathbf{\Delta})$ propyl acetate, isopropyl acetate, ( ) butyl acetate)(1)+olive oil (2) and (一, Eq. 3) fitting curves

Mixtures containing esters as well as higher molecules (nnonane, butyl acetate) gather the best results in terms of deviations (Figure 4). Experimental data of mixing ultrasonic velocities were compared to values obtained by the following rules: Danusso (eq. 14) [28], Nomoto (eq. 15) [29], Junjie (eq. 16) [30], Impedance Model (eq. 17) [31], Collision Factor Theory (eq. 18) [32], and Free Length Theory (eq. 19) [33].

$$
u=\frac{1}{\rho_{\text {mix }}}\left(\frac{1}{M_{e f f}} \sum \frac{x_{i} M_{i}}{\rho_{i}^{2} u_{i}^{2}}\right)^{1 / 2}
$$

where $\mathrm{M}_{\text {eff }}$ is the effective molar mass.

$$
u=\left(\sum \frac{x_{i} R_{i}}{x_{i} V_{i}}\right)^{3}
$$

where $\mathrm{R}$ and $\mathrm{V}$ are molar sound velocity and molar volume. 
Table 5. Experimental data of ultrasonic velocity, isentropic compressibilities and change of isentropic compressibilities for esters + olive oil mixtures at 288.15, 293.15 and 298.15 K.

\begin{tabular}{|c|c|c|c|c|c|c|c|c|c|}
\hline \multicolumn{4}{|c|}{$\mathrm{u} / \mathrm{m} \cdot \mathrm{s}^{-1}$} & \multicolumn{3}{|c|}{$\kappa_{\mathrm{S}} / \mathrm{TPa}^{-1}$} & \multicolumn{3}{|c|}{$\delta \kappa_{\mathrm{s}} / \mathrm{TPa}^{-1}$} \\
\hline $\mathrm{X}_{1}$ & 298.15 & 293.15 & 288.15 & 298.15 & 293.15 & 288.15 & 298.15 & 293.15 & 288.15 \\
\hline \multicolumn{10}{|c|}{ Ethyl Acetate + Olive oil } \\
\hline 0.0466 & 1446.0 & 1463.0 & 1480.6 & 526.3 & 512.2 & 498.2 & -14.2 & -13.1 & -12.3 \\
\hline 0.0951 & 1443.5 & 1460.5 & 1477.9 & 528.1 & 514.0 & 500.0 & -28.7 & -26.4 & -24.4 \\
\hline 0.1904 & 1437.8 & 1454.8 & 1472.3 & 532.5 & 518.1 & 504.0 & -56.4 & -52.0 & -48.1 \\
\hline 0.2997 & 1430.1 & 1447.2 & 1464.5 & 538.4 & 523.8 & 509.5 & -87.2 & -80.4 & -74.2 \\
\hline 0.3963 & 1421.1 & 1438.4 & 1455.9 & 545.5 & 530.4 & 515.7 & -112.7 & -103.9 & -95.9 \\
\hline 0.4478 & 1415.3 & 1432.9 & 1450.2 & 550.1 & 534.6 & 519.9 & -125.3 & -115.8 & -106.6 \\
\hline 0.5007 & 1408.6 & 1426.2 & 1443.7 & 555.5 & 539.7 & 524.7 & -137.8 & -127.1 & -117.1 \\
\hline 0.5557 & 1400.3 & 1418.1 & 1435.5 & 562.3 & 546.1 & 530.9 & -149.4 & -137.9 & -126.9 \\
\hline 0.6025 & 1391.9 & 1409.9 & 1427.7 & 569.3 & 552.6 & 536.8 & -158.2 & -145.9 & -134.5 \\
\hline 0.7022 & 1368.6 & 1387.0 & 1405.0 & 589.5 & 571.6 & 554.7 & -171.5 & -158.0 & -145.4 \\
\hline 0.8089 & 1329.2 & 1349.2 & 1368.1 & 626.2 & 604.9 & 585.8 & -170.7 & -157.9 & -145.2 \\
\hline 0.8989 & 1271.6 & 1291.6 & 1311.4 & 686.1 & 661.8 & 638.7 & -141.1 & -129.1 & -118.3 \\
\hline 0.9519 & 1215.7 & 1236.8 & 1257.9 & 753.0 & 723.5 & 695.6 & -92.1 & -84.0 & -76.8 \\
\hline \multicolumn{10}{|c|}{ Vinyl Acetate + Olive Oil } \\
\hline 0.0435 & 1446.2 & 1463.1 & 1480.2 & 526.0 & 512.0 & 498.3 & -13.6 & -12.4 & -11.5 \\
\hline 0.1001 & 1443.2 & 1460.1 & 1477.2 & 528.1 & 514.0 & 500.3 & -30.9 & -28.3 & -26.2 \\
\hline 0.2079 & 1436.4 & 1453.6 & 1470.2 & 533.1 & 518.5 & 505.0 & -62.9 & -58.1 & -53.3 \\
\hline 0.3041 & 1429.1 & 1446.4 & 1463.5 & 538.4 & 523.5 & 509.4 & -90.6 & -83.7 & -77.2 \\
\hline 0.3956 & 1420.3 & 1437.6 & 1454.8 & 544.9 & 529.9 & 515.4 & -115.5 & -106.5 & -98.2 \\
\hline 0.4418 & 1415.2 & 1432.5 & 1449.7 & 548.8 & 533.5 & 518.9 & -127.5 & -117.5 & -108.3 \\
\hline 0.5072 & 1406.3 & 1423.8 & 1441.1 & 555.6 & 539.9 & 524.9 & -143.1 & -132.0 & -121.7 \\
\hline 0.5433 & 1400.1 & 1417.8 & 1435.1 & 560.4 & 544.3 & 529.2 & -150.7 & -139.0 & -128.0 \\
\hline 0.5973 & 1390.6 & 1408.3 & 1425.7 & 567.9 & 551.5 & 535.9 & -161.7 & -149.0 & -137.2 \\
\hline 0.7053 & 1365.0 & 1383.1 & 1400.9 & 588.9 & 571.1 & 554.3 & -177.8 & -163.7 & -150.6 \\
\hline 0.7968 & 1328.0 & 1347.6 & 1366.0 & 621.2 & 600.6 & 581.9 & -176.9 & -163.4 & -150.0 \\
\hline 0.8972 & 1260.1 & 1279.7 & 1299.3 & 687.8 & 663.4 & 640.4 & -144.8 & -132.4 & -121.2 \\
\hline 0.9605 & 1199.2 & 1219.9 & 1240.5 & 756.7 & 727.0 & 699.1 & -97.6 & -89.0 & -81.1 \\
\hline \multicolumn{10}{|c|}{ Propyl Acetate + Olive Oil } \\
\hline 0.0451 & 1446.1 & 1463.1 & 1480.9 & 526.2 & 512.1 & 498.0 & -12.6 & -11.7 & -11.1 \\
\hline 0.0914 & 1443.7 & 1460.8 & 1477.9 & 528.1 & 513.8 & 500.1 & -25.0 & -23.2 & -21.4 \\
\hline 0.1987 & 1437.4 & 1454.7 & 1471.6 & 533.0 & 518.4 & 504.7 & -53.4 & -49.5 & -45.7 \\
\hline 0.3039 & 1429.7 & 1446.9 & 1464.1 & 539.1 & 524.3 & 510.1 & -80.0 & -73.9 & -68.6 \\
\hline 0.3903 & 1421.9 & 1438.9 & 1456.5 & 545.4 & 530.5 & 515.7 & -100.4 & -92.5 & -86.1 \\
\hline 0.4476 & 1415.6 & 1432.7 & 1450.4 & 550.6 & 535.4 & 520.4 & 13.0 & -104.1 & -96.9 \\
\hline 0.4963 & 1409.6 & 14 & 1444.5 & 555.6 & 540.1 & 524.9 & 23.1 & -113.4 & -105.5 \\
\hline 0.5412 & 1403.1 & 1420.5 & 1438.3 & 561.0 & 545.2 & 529.6 & -131.6 & -121.2 & -112.8 \\
\hline 0.6020 & 1392.8 & 1410.2 & 1428.3 & 569.9 & 553.6 & 537.5 & -141.6 & -130.3 & -121.3 \\
\hline 0.7001 & 1370.9 & 1388.7 & 1407.0 & 589.3 & 571.9 & 554.8 & -152.6 & -140.2 & -130.4 \\
\hline 0.8018 & 1336.4 & 1354.2 & 1373.6 & 622.1 & 603.2 & 583.7 & -151.3 & -138.2 & -128.8 \\
\hline 0.9001 & 1279.7 & 1298.6 & 1318.8 & 682.4 & 659.5 & 636.2 & -121.5 & -110.2 & -102.7 \\
\hline 0.9517 & 1231.7 & 1252.2 & 1272.7 & 740.6 & 712.7 & 686.3 & -79.2 & -71.7 & -66.5 \\
\hline \multicolumn{10}{|c|}{ Isopropyl Acetate + Olive Oil } \\
\hline 0.0489 & 1445.3 & 1462.0 & 1479.4 & 526.8 & 512.9 & 499.1 & \begin{tabular}{|l|}
-18.6 \\
\end{tabular} & -17.1 & -15.8 \\
\hline 0.1005 & 1442.2 & 1458.7 & 1476.2 & 529.3 & 515.4 & 501.5 & -38.0 & -35.0 & -32.4 \\
\hline 0.2033 & 1434.6 & 1451.3 & 1468.9 & 535.4 & 521.1 & 506.8 & -75.4 & -69.8 & -64.8 \\
\hline & 1425.7 & 14 & 8 & .5 & 528.1 & 6 & 10.6 & -102.3 & -94.8 \\
\hline & 1414.8 & & & & 536.6 & 521.5 & -142.6 & -132.1 & -122.6 \\
\hline 0.4446 & 1409.0 & 1425.8 & 1443.7 & 556.5 & 541.3 & 526.0 & -156.3 & -144.7 & -134.2 \\
\hline 0.4946 & 1401.4 & 1418.4 & 1436.3 & 562.9 & 547.4 & 531.7 & -171.0 & -158.3 & -146.8 \\
\hline 0.5504 & 1391.7 & 1408.7 & 1426.7 & 571.5 & 555.5 & 539.5 & -186.1 & -172.2 & -159.6 \\
\hline 0.5966 & 1382.2 & 1399.3 & 1417.4 & 579.9 & 563.6 & 547.1 & -197.1 & -182.3 & -168.9 \\
\hline 0.6947 & 1356.1 & 1373.6 & 1392.0 & 604.2 & 586.5 & 568.7 & -214.3 & -198.1 & -183.3 \\
\hline 0.7971 & 1314.5 & 1332.6 & 1351.6 & 646.3 & 626.0 & 605.9 & -215.5 & -198.9 & -183.7 \\
\hline 0.8988 & 1244.2 & 1263.5 & 1283.5 & 728.0 & 702.5 & 677.4 & -176.8 & -162.5 & -149.5 \\
\hline 0.9558 & 1178.5 & 1199.1 & 1220.2 & 819.7 & 787.3 & 756.1 & -109.2 & -100.2 & -91.8 \\
\hline \multicolumn{10}{|c|}{ Butyl Acetate + Olive Oil } \\
\hline 0.0503 & 1445.8 & 1462.4 & 1479.9 & 526.5 & 512.7 & 498.7 & -12.5 & -11.4 & -10.6 \\
\hline 0.0995 & 1443.3 & 1459.9 & 1477.5 & 528.4 & 514.5 & 500.5 & -24.4 & -22.5 & -21.0 \\
\hline 0.1896 & 1438.1 & 1454.8 & 1472.4 & 532.6 & 518.4 & 504.2 & -45.5 & -42.3 & -39.5 \\
\hline 0.3056 & 1429.9 & 1446.6 & 1464.3 & 539.2 & 524.8 & 510.3 & -71.4 & -66.4 & -62.0 \\
\hline 0.3939 & 1422.2 & 1438.9 & 1456.6 & 545.5 & 530.9 & 516.1 & -89.9 & -83.6 & -77.9 \\
\hline 0.4435 & 1416.9 & 1433.8 & 1451.6 & 550.0 & 535.0 & 520.0 & -99.4 & -92.5 & -86.3 \\
\hline 0.4972 & 1410.5 & 1427.4 & 1445.2 & 555.3 & 540.2 & 524.9 & -109.0 & -101.5 & -94.5 \\
\hline 0.5603 & 1401.7 & 1418.7 & 1436.6 & 563.0 & 547.5 & 531.8 & -119.2 & -110.8 & -103.3 \\
\hline 0.6002 & 1395.2 & 1412.1 & 1430.2 & 568.7 & 552.9 & 536.9 & -124.7 & -115.8 & -108.0 \\
\hline 0.6987 & 1374.5 & 1391.6 & 1410.1 & 587.3 & 570.7 & 553.6 & -133.6 & -124.0 & -115.5 \\
\hline 0.8108 & 1338.3 & 1356.2 & 1375.0 & 622.4 & 603.5 & 584.6 & -130.0 & -120.7 & -112.2 \\
\hline 0.9076 & 1285.4 & 1304.1 & 1323.7 & 679.7 & 657.3 & 635.0 & -99.9 & -92.4 & -85.6 \\
\hline 0.9492 & 1250.5 & 1269.9 & 1290.0 & 722.1 & 696.7 & 671.7 & -69.2 & -64.0 & -59.1 \\
\hline
\end{tabular}

$$
\begin{aligned}
& u=\left(\sum \frac{x_{i} V_{i}}{x_{i} M_{i}}\right)^{1 / 2}\left(\sum \frac{x_{i} V_{i}}{\rho_{i} u_{i}}\right)^{-1 / 2} \\
& u=\left(\frac{\sum x_{i} Z_{i}}{\sum x_{i} \rho_{i}}\right)^{-12}
\end{aligned}
$$
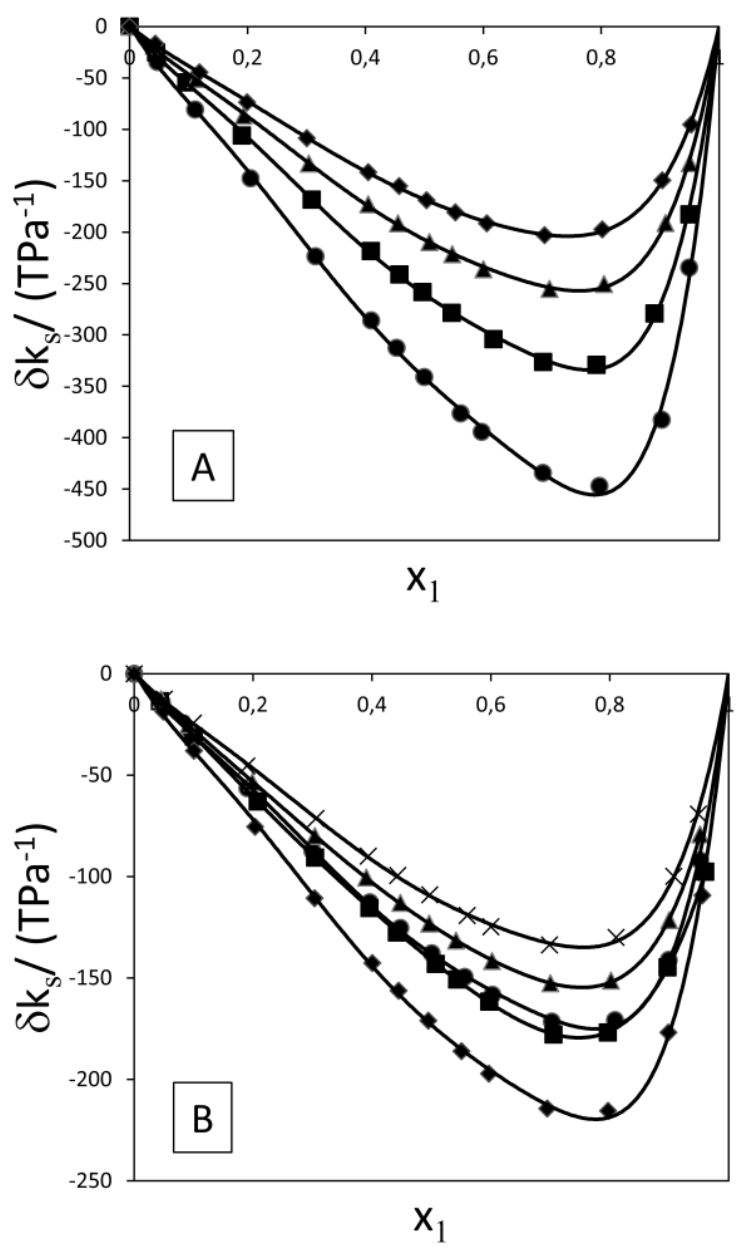

Figure 2. Change of isentropic compressibilities $\left(\mathrm{TPa}^{-1}\right)$ versus mole fraction at $298.15 \mathrm{~K}$ for $(A)((\bullet) n$-hexane,

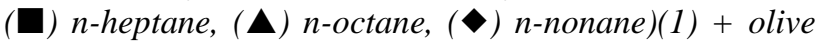
oil (2) and (- Eq. 3) fitting curves and for $(B)((\bullet)$ ethyl acetate, $(*)$ vinyl actetate, ( $\mathbf{\Delta})$ propyl acetate, isopropyl acetate, $(\bullet)$ butyl acetate $)(1)+$ olive oil (2) and (- Eq. 3) fitting curves

The Collision Factor Theory (CFT) is dependent on the collision factors among molecules as a function of temperature into pure solvent or mixture (eq. 18). The collision factors $(\mathrm{S})$ and the characteristic molecular volumes (B) of the pure solvents used in the CFT calculations were estimated by using the experimental ultrasonic velocities, enclosed in this paper, and the corresponding molar volumes from open literature [1617]. These values could be also evaluated by means a group contribution procedure (Bondi's group contribution methodology), when no experimental ones are disposable.

$u=\frac{u_{\infty} \sum x_{i} S_{i} \sum x_{i} B_{i}}{V_{\text {mix }}}$

In this equation, $\mathrm{u}_{\infty}$ is $1600 \mathrm{~m} / \mathrm{s}, \mathrm{S}$ is collision factor, $\mathrm{B}$ is volume of molecule per mole and $\mathrm{V}$ is molar volume. The Free Length Theory (FLT) estimates the ultrasonic velocity of a mixture attending to the free displacement of molecules $\left(\mathrm{L}_{\mathrm{f}}\right)$ as a main function of temperature, then (eq. 19):

where $Z_{i}$ is specific acoustic impedance. 


$$
u=\frac{K}{L_{\text {mix }} \rho_{\text {mix }}^{1 / 2}}
$$

where

$$
L_{\text {mix }}=2\left(\frac{V_{m i x}-\sum x_{i} V_{i}}{\sum x_{i} Y_{i}}\right)
$$

where $V_{i}$ represent the volume at absolute zero of each pure component and $\mathrm{Y}_{\mathrm{i}}$ is the surface area per mole, and is defined as:

$$
Y=\frac{2 V_{\text {mix }}}{L_{f_{\text {mix }}}}\left(1-\frac{u_{\text {exp }}}{u_{\infty}}\right)
$$

The pertinent relations in these theories, as well as, their theoretical basis were described at the literature cited.

\section{Discussion and conclusions}

Although the mixing properties behaviour as a function of temperature or pressure has been investigated in the last few years, most studies have been related to liquid mixtures containing light molecules, biological macromolecules has hitherto received comparatively little attention. This fact is really amazing attending to its key role in food industry or biotechnology processes. In spite of much investigation, however, the exact nature of solvation procedure of macromolecules in short solvents, as triglycerides in $\mathrm{n}$-alkane or ester solvents, remains a little bit obscure. Up to the present, little attention has been paid to thermodynamic studies in edible oil mixtures with potential separation solvents by environment friendly procedures of extraction, and refining such as modified distillation or wintering.

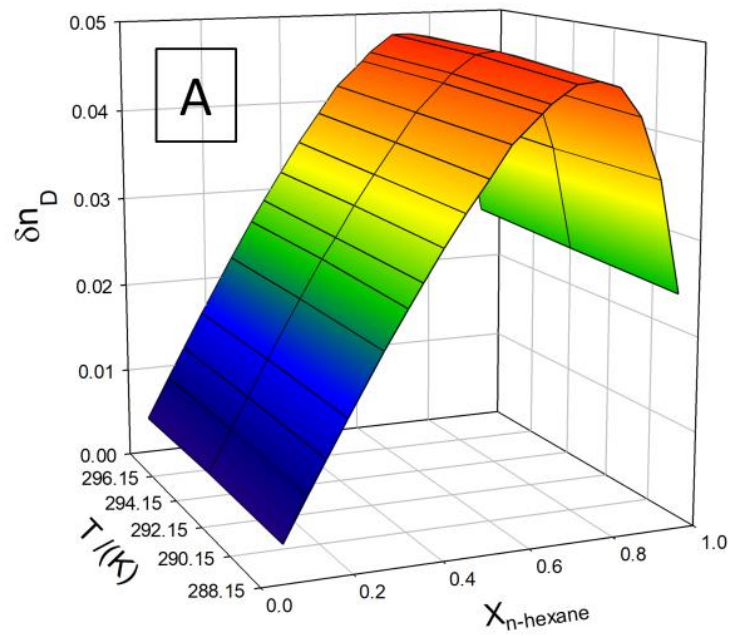

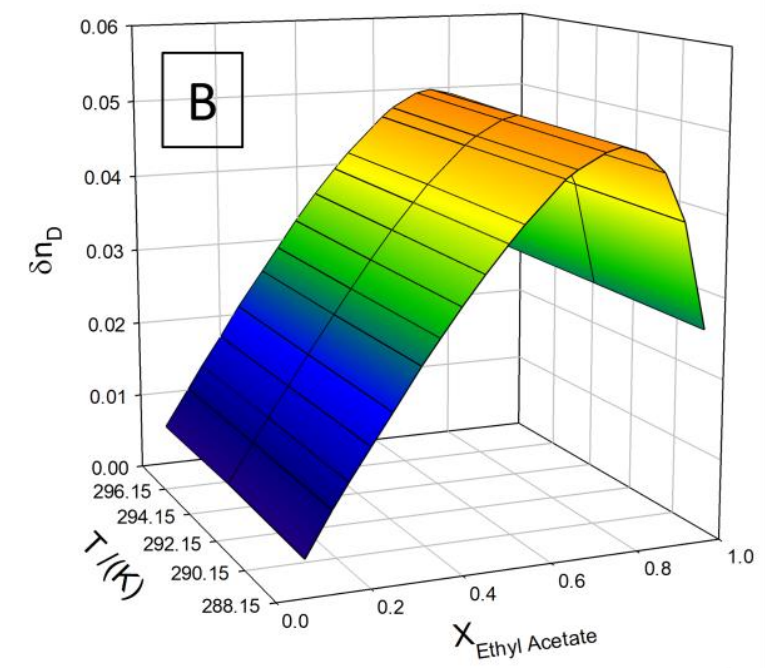
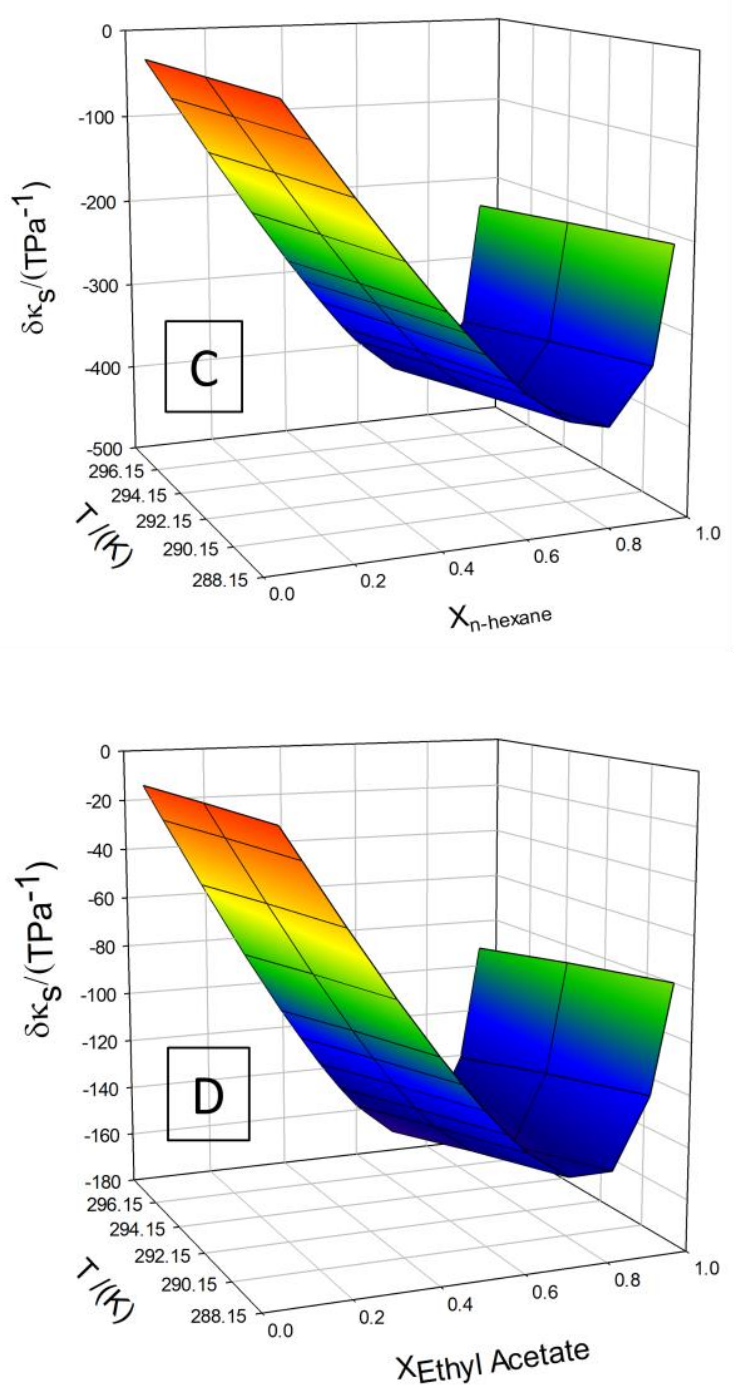

Figure 3. Surfaces of changes of refractive index on mixing of ((A) n-hexane and $(B)$ ethyl acetate $))+$ olive oil mixtures at the range of temperatures 288.15-298.15 $K$ and surfaces of changes of isentropic compressibilities of ((C) n-hexane and (D) ethyl acetate)) + olive oil mixtures at the range of temperatures 288.15-298.15 K 


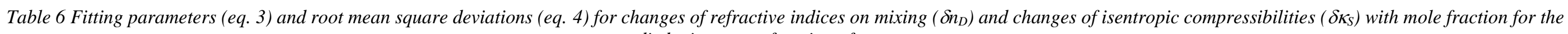
studied mixtures as function of temperature.

\begin{tabular}{|c|c|c|c|c|c|c|c|c|c|c|c|c|}
\hline $\begin{array}{l}\text { Derived } \\
\text { Property }\end{array}$ & $B_{00}$ & $B_{01}$ & $B_{02}$ & $B_{10}$ & $B_{11}$ & $B_{12}$ & $B_{20}$ & $B_{21}$ & $B_{22}$ & $B_{30}$ & $B_{31}$ & $B_{32}$ \\
\hline
\end{tabular}

n-Hexane + Olive Oil

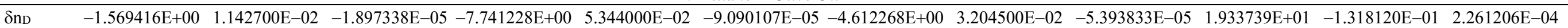
$\sigma=9.0 \mathrm{E}-04$

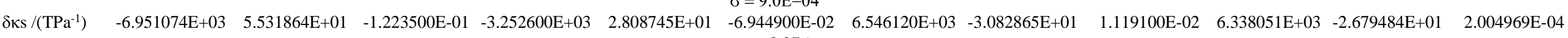
$\sigma=8.276$

n-Heptane + Olive Oil

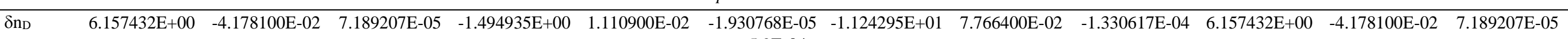
$\sigma=5.9 \mathrm{E}-04$

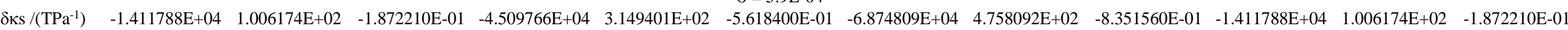
$\sigma=6.210$

n-Octane + Olive Oil

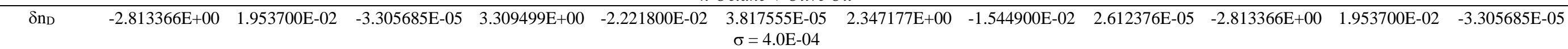

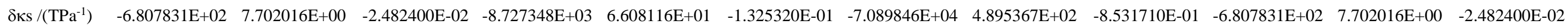
$\sigma=3.989$

n-Nonane + Olive Oil

\begin{tabular}{|c|c|c|c|c|c|c|c|c|c|c|c|c|}
\hline & & & & & & & & & & & & \\
\hline$\delta \mathrm{n}_{\mathrm{D}}$ & $-3.268780 \mathrm{E}-01$ & $2.560954 \mathrm{E}-03$ & $-4.224202 \mathrm{E}-06$ & $-8.175710 \mathrm{E}-01$ & $5.878134 \mathrm{E}-03$ & $\begin{array}{c}-9.838215 \mathrm{E}-06 \\
\sigma=2.4 \mathrm{E}-04\end{array}$ & $-3.880076 \mathrm{E}+00$ & $2.669700 \mathrm{E}-02$ & $-4.537785 \mathrm{E}-05$ & $-3.268780 \mathrm{E}-01$ & $2.560954 \mathrm{E}-03$ & $-4.224202 \mathrm{E}-06$ \\
\hline$\delta \kappa s /\left(\mathrm{TPa}^{-1}\right)$ & $1.030563 \mathrm{E}+04$ & $-6.788977 \mathrm{E}+01$ & $1.064190 \mathrm{E}-01$ & $1.234711 \mathrm{E}+04$ & $-8.053830 \mathrm{E}+01$ & $\begin{array}{c}1.247040 \mathrm{E}-01 \\
\sigma=2.628\end{array}$ & $-3.202516 \mathrm{E}+04$ & $2.208822 \mathrm{E}+02$ & $-3.864310 \mathrm{E}-01$ & $1.030563 \mathrm{E}+04$ & $-6.788977 \mathrm{E}+01$ & $1.064190 \mathrm{E}-01$ \\
\hline
\end{tabular}

Ethyl Acetate + Olive Oil

\begin{tabular}{|c|c|c|c|c|c|c|c|c|c|c|c|c|}
\hline \multicolumn{13}{|c|}{ Ethyl Acetate + Olive Oil } \\
\hline$\delta \mathrm{n}_{\mathrm{D}}$ & $-2.745930 \mathrm{E}+00$ & $1.932800 \mathrm{E}-02$ & $-3.266165 \mathrm{E}-05$ & $-7.086320 \mathrm{E}-01$ & 5.398762E-03 & $\begin{array}{c}-8.285304 \mathrm{E}-06 \\
\sigma=1.2 \mathrm{E}-03\end{array}$ & $1.085279 \mathrm{E}+01$ & $-7.313200 \mathrm{E}-02$ & $1.250593 \mathrm{E}-04$ & $-2.745930 \mathrm{E}+00$ & $1.932800 \mathrm{E}-02$ & $-3.266165 \mathrm{E}-05$ \\
\hline$\delta \kappa s /\left(\mathrm{TPa}^{-1}\right)$ & $3.009511 \mathrm{E}+03$ & $-1.727985 \mathrm{E}+01$ & $1.954300 \mathrm{E}-02$ & $-8.247765 \mathrm{E}+03$ & $6.233105 \mathrm{E}+01$ & $\begin{array}{c}-1.229430 \mathrm{E}-01 \\
\sigma=3.037\end{array}$ & $-9.837252 \mathrm{E}+03$ & $7.412555 \mathrm{E}+01$ & $-1.443380 \mathrm{E}-01$ & $3.009511 \mathrm{E}+03$ & $-1.727985 \mathrm{E}+01$ & $1.954300 \mathrm{E}-02$ \\
\hline \multicolumn{13}{|c|}{ Vinyl Acetate + Olive Oil } \\
\hline$\delta n_{D}$ & $-4.604430 \mathrm{E}-01$ & $3.432079 \mathrm{E}-03$ & $-5.318987 \mathrm{E}-06$ & $-7.398330 \mathrm{E}-01$ & $5.671864 \mathrm{E}-03$ & $\begin{array}{c}-9.232118 \mathrm{E}-06 \\
\sigma=9.4 \mathrm{E}-04\end{array}$ & $1.856989 \mathrm{E}+00$ & $-1.194900 \mathrm{E}-02$ & $2.062344 \mathrm{E}-05$ & $-4.604430 \mathrm{E}-01$ & $3.432079 \mathrm{E}-03$ & $-5.318987 \mathrm{E}-06$ \\
\hline$\delta \kappa s /\left(\mathrm{TPa}^{-1}\right)$ & $-8.312572 \mathrm{E}+02$ & $8.940438 \mathrm{E}+00$ & $-2.510900 \mathrm{E}-02$ & $-3.152335 \mathrm{E}+03$ & $2.843911 \mathrm{E}+01$ & $\begin{array}{c}-6.704700 \mathrm{E}-02 \\
\sigma=5.631\end{array}$ & $-3.568747 \mathrm{E}+03$ & $3.238902 \mathrm{E}+01$ & $-7.602100 \mathrm{E}-02$ & $-8.312572 \mathrm{E}+02$ & $8.940438 \mathrm{E}+00$ & $-2.510900 \mathrm{E}-02$ \\
\hline
\end{tabular}

Propyl Acetate + Olive Oil

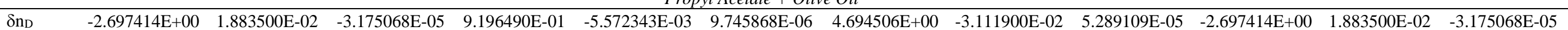

100 / Vol. 23 (No. 2)

Int. Centre for Applied Thermodynamics (ICAT) 
$\sigma=8.1 \mathrm{E}-04$

$\begin{array}{lllllll} & -4.454868 \mathrm{E}+04 & 3.097128 \mathrm{E}+02 & -5.428890 \mathrm{E}-01 & -1.178494 \mathrm{E}+04 & 8.316042 \mathrm{E}+01 & -1.503910 \mathrm{E}-01\end{array}$ $\delta \kappa s /\left(\mathrm{TPa}^{-1}\right) \quad-1.178494 \mathrm{E}+04 \quad 8.316042 \mathrm{E}+01 \quad-1.503910 \mathrm{E}-01 \quad-2.979639 \mathrm{E}+04 \quad 2.085153 \mathrm{E}+02 \quad-3.697840 \mathrm{E}-01$

Isopropyl Acetate + Olive Oil

\begin{tabular}{|c|c|c|c|c|c|c|c|c|c|c|c|c|}
\hline \multicolumn{13}{|c|}{ Isopropyl Acetate + Olive Oil } \\
\hline$\delta \mathrm{nD}_{\mathrm{D}}$ & $2.189125 \mathrm{E}+00$ & $-1.448800 \mathrm{E}-02$ & $2.514124 \mathrm{E}-05$ & $3.591805 \mathrm{E}+00$ & $-2.387600 \mathrm{E}-02$ & $\begin{array}{c}4.120955 \mathrm{E}-05 \\
\sigma=8.9 \mathrm{E}-04\end{array}$ & $-6.160423 \mathrm{E}+00$ & $4.292900 \mathrm{E}-02$ & $-7.328615 \mathrm{E}-05$ & $2.189125 \mathrm{E}+00$ & $-1.448800 \mathrm{E}-02$ & $2.514124 \mathrm{E}-05$ \\
\hline$\delta \kappa s /\left(\mathrm{TPa}^{-1}\right)$ & $-5.800561 \mathrm{E}+03$ & $4.185520 \mathrm{E}+01$ & $-7.867400 \mathrm{E}-02$ & $-5.528279 \mathrm{E}+03$ & $4.152651 \mathrm{E}+01$ & $\begin{array}{c}-8.178000 \mathrm{E}-02 \\
\sigma=1.963\end{array}$ & $7.910274 \mathrm{E}+03$ & $-5.058529 \mathrm{E}+01$ & 7.644500E-02 & $-5.800561 \mathrm{E}+03$ & $4.185520 \mathrm{E}+01$ & $-7.867400 \mathrm{E}-02$ \\
\hline \multicolumn{13}{|c|}{ Butyl Acetate + Olive Oil } \\
\hline$\delta \mathrm{n}_{\mathrm{D}}$ & $7.170990 \mathrm{E}-01$ & $-4.458244 \mathrm{E}-03$ & 7.797277E-06 & $2.413420 \mathrm{E}+00$ & $-1.588900 \mathrm{E}-02$ & $\begin{array}{c}2.723102 \mathrm{E}-05 \\
\sigma=5.7 \mathrm{E}-04\end{array}$ & $-2.365500 \mathrm{E}-02$ & $6.894787 \mathrm{E}-04$ & $-1.092955 \mathrm{E}-06$ & $7.170990 \mathrm{E}-01$ & $-4.458244 \mathrm{E}-03$ & 7.797277E-06 \\
\hline$\delta \kappa s /\left(\mathrm{TPa}^{-1}\right)$ & $-3.252600 \mathrm{E}+03$ & $2.808745 \mathrm{E}+01$ & $-6.944900 \mathrm{E}-02$ & $6.546120 \mathrm{E}+03$ & $-3.082865 \mathrm{E}+01$ & $\begin{array}{c}1.119100 \mathrm{E}-02 \\
\sigma=8.276\end{array}$ & $6.338051 \mathrm{E}+03$ & $-2.679484 \mathrm{E}+01$ & $2.004969 \mathrm{E}-04$ & $-3.252600 \mathrm{E}+03$ & $2.808745 \mathrm{E}+01$ & $-6.944900 \mathrm{E}-02$ \\
\hline
\end{tabular}


Table 7. Root mean square deviations of experimental results from the estimated results for refractive indices on mixing by mixing rules (eqs. 5-13) at $298.15 \mathrm{~K}$.

(eq. 5) (eq.6) (eq.7) (eq. 8) (eq.9) (eq. 10) (eq. 11) (eq. 12) (eq. 13)

Additivity on Mixing

$n$-Hexane $0.00403 \quad 0.004340 .004250 .004340 .004670 .00453 \quad 0.004190 .011420 .00338$ $n$-Heptane 0.001840 .001490 .001600 .001490 .001160 .001300 .001660 .004220 .00207

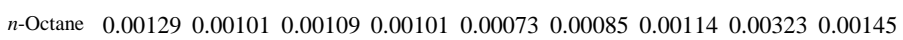

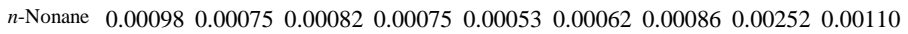
$\begin{array}{llllllllll}\text { Ethyl } & 0.00028 & 0.00021 & 0.00011 & 0.00021 & 0.00063 & 0.00045 & 0.00011 & 0.00785 & 0.00102\end{array}$ Acetate

$\begin{array}{lllllllllll}\text { Vinyl } & 0.00012 & 0.00033 & 0.00026 & 0.00033 & 0.00058 & 0.00047 & 0.00021 & 0.00467 & 0.00054\end{array}$ Acetate

Propyl $\quad 0.000350 .000090 .000120 .000090 .000390 .000240 .00017 \quad 0.005790 .00082$ Acetate

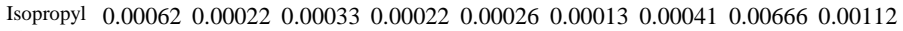
Acetate

$\begin{array}{lllllllllll}\text { Butyl } & 0.00035 & 0.00008 & 0.00015 & 0.00008 & 0.00023 & 0.00011 & 0.00021 & 0.00438 & 0.00060\end{array}$ Acetate

$$
\text { Non Additivity on Mixing }
$$

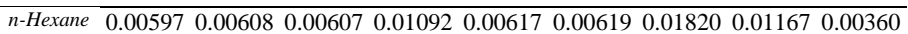

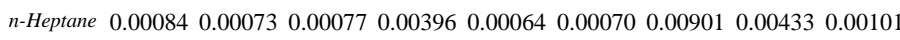

$\begin{array}{llllllllll}n \text {-Octane } & 0.00067 & 0.00054 & 0.00058 & 0.00251 & 0.00045 & 0.00049 & 0.00568 & 0.00328 & 0.00137\end{array}$

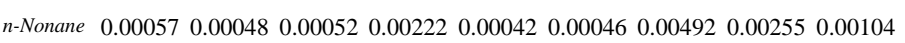

$\begin{array}{llllllllll}\text { Ethyl } & 0.00066 & 0.00026 & 0.00037 & 0.00098 & 0.00043 & 0.00027 & 0.00227 & 0.00779 & 0.00107\end{array}$

$\begin{array}{lllllllllll}\text { Vinyl } & 0.00049 & 0.00028 & 0.00033 & 0.00112 & 0.00035 & 0.00028 & 0.00257 & 0.00461 & 0.00058\end{array}$

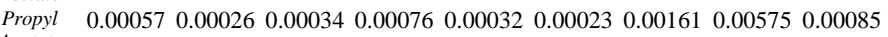

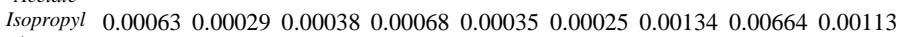

Acetate

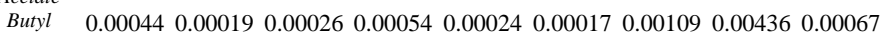
Acetate

Table 8. Root mean square deviations (eq. 4) of the experimental and estimated results for ultrasonic velocities

$\left(\mathrm{m} \cdot \mathrm{s}^{-1}\right)$ by different models (eqs. 14-19) at $298.15 \mathrm{~K}$.

\begin{tabular}{lcccccc}
\hline & Danusso & Nomoto & Junjie & $\begin{array}{c}\text { Impedance } \\
\text { Model }\end{array}$ & CFT & FLT \\
& (eq. 14) & (eq. 15) & (eq. 16) & $\begin{array}{c}\text { (eq. 17) } \\
\text { (eq. 18) }\end{array}$ & (eq. 19) \\
\hline$n$-Hexane & 47.53 & 5.36 & 42.25 & 87.01 & 86.51 & 91.66 \\
$n$-Heptane & 29.21 & 2.85 & 31.74 & 72.2 & 57.65 & 82.55 \\
$n$-Octane & 23.31 & 2.94 & 24.01 & 59.3 & 51.02 & 83.63 \\
$n$-Nonane & 25.33 & 3.09 & 18.71 & 49.69 & 41.97 & 81.06 \\
Ethyl Acetate & 23.03 & 11.59 & 0.81 & 90.02 & 60.82 & 96.28 \\
Vinyl Acetate & 17.33 & 12.01 & 2.50 & 100.66 & 70.32 & 92.23 \\
Propyl Acetate & 25.80 & 8.25 & 2.54 & 78.62 & 51.81 & 90.48 \\
Isopropyl Acetate & 6.75 & 8.32 & 8.82 & 95.05 & 77.78 & 95.86 \\
Butyl Acetate & 29.52 & 5.64 & 3.85 & 68.96 & 45.55 & 84.27 \\
\hline
\end{tabular}

These mixtures show a high non-ideal trend which is severe conditioned by two phenomenon; the associative behaviour by dispersive forces among aliphatic ends and polar interactions among polar groups (ester functional groups) and the huge difference in molecular volume between each solvent and triglyceride (non polar flexible chains) that leads to important steric hyndrance effects. These factors, under mixing conditions, produce a) variation of the intermolecular forces and b) variation of molecular packing as a consequence of the size and shape differences between components, providing considerable non-ideality in this type of mixtures, and clearly two different trends. Firstly, solvents of aliphatic nature show contractive trend, inversely related to increasing chain size and directly related to rising temperatures (higher contraction for $n$ hexane that for $n$-nonane and stronger contraction for rising values of temperature), which is reflected as positive deviations in terms of change of refractive indices on mixing and strong negative deviations of change of isentropic compressibilities (Figures 1A and 2A). This tendency is coherent with earlier studies of this kind of mixtures [16] due to the easy packing of paraffinic chains into triglyceride molecules, in general terms.
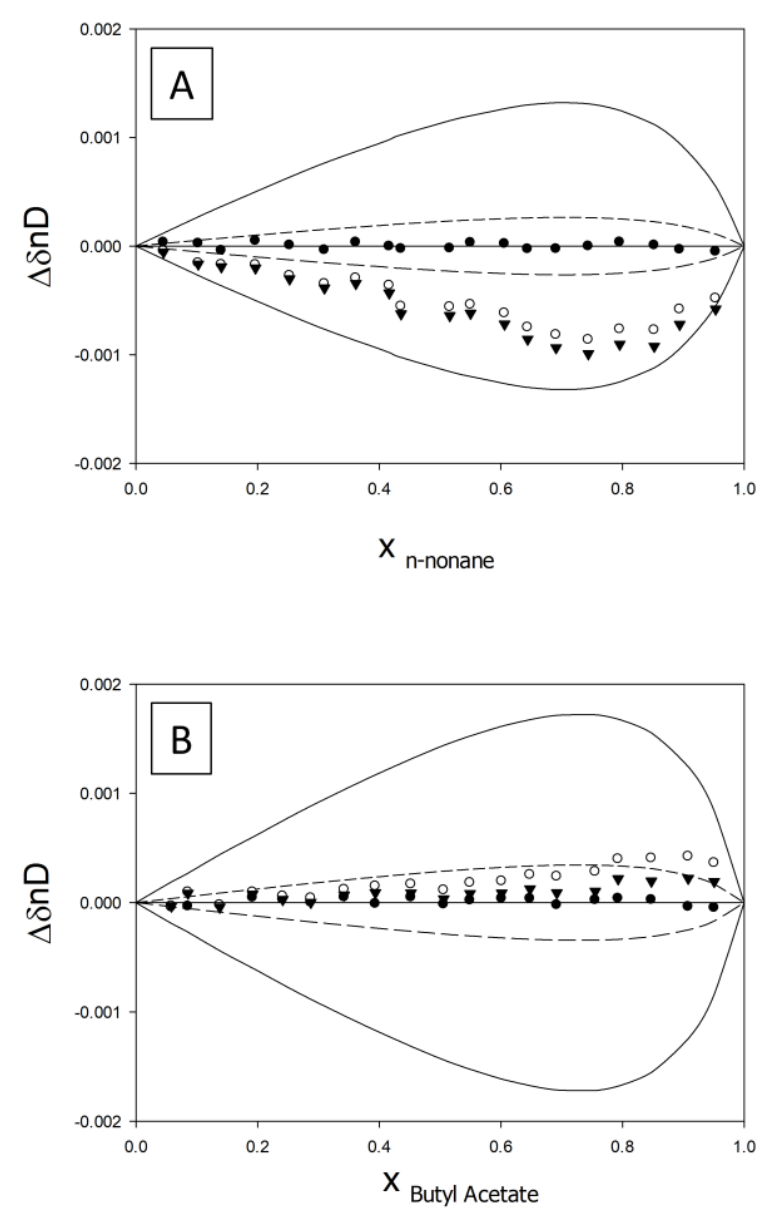

Fig. 4. Comparison of experimental and estimated data

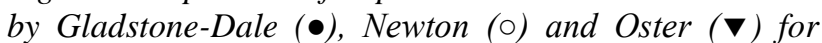
change of refractive index on mixing at $298.15 \mathrm{~K}$, for ((a) n-nonane and (b) butyl acetate) + olive oil mixtures. The lines (----, 1\% deviation; - $5 \%$ deviation) indicate percentual deviation from experimental values as a reference.

As previously observed, pure paraffinic chains as nalkane compounds are easily introduced among bulky hydrophobic side of glycerides mixing molecular volumes resulting in strong contractive trend mainly at the higher studied temperatures. These facts reveal as 1) higher temperature levels allow to access close empty spaces around macromolecules due to a higher molecular kinetics and 2) relative short paraffinic chains achieve more efficient disperse interaction without high level of steric hindrance (see comparison of n-nonane and nhexane in terms of $\delta n_{D}$ or excess molar volume [34]. Secondly, for mixtures containing esters and olive oil, 
low temperatures deals for expansive trend, only isopropyl and butyl acetate mixtures showing contractive tendence at the highest temperatures. These trend are reflected too in terms of the studied properties change of refractive indices on mixing (positive values) and change of isentropic compressibilities (moderate negative values) (Figures 1B and 2B). For comparison, the evolution of $n$ hexane and ethyl acetate + olive oil are gathered as a funtion of composition and temperature in Figure 3 for both change of refractive index on mixing and change of isentropic compressibility.
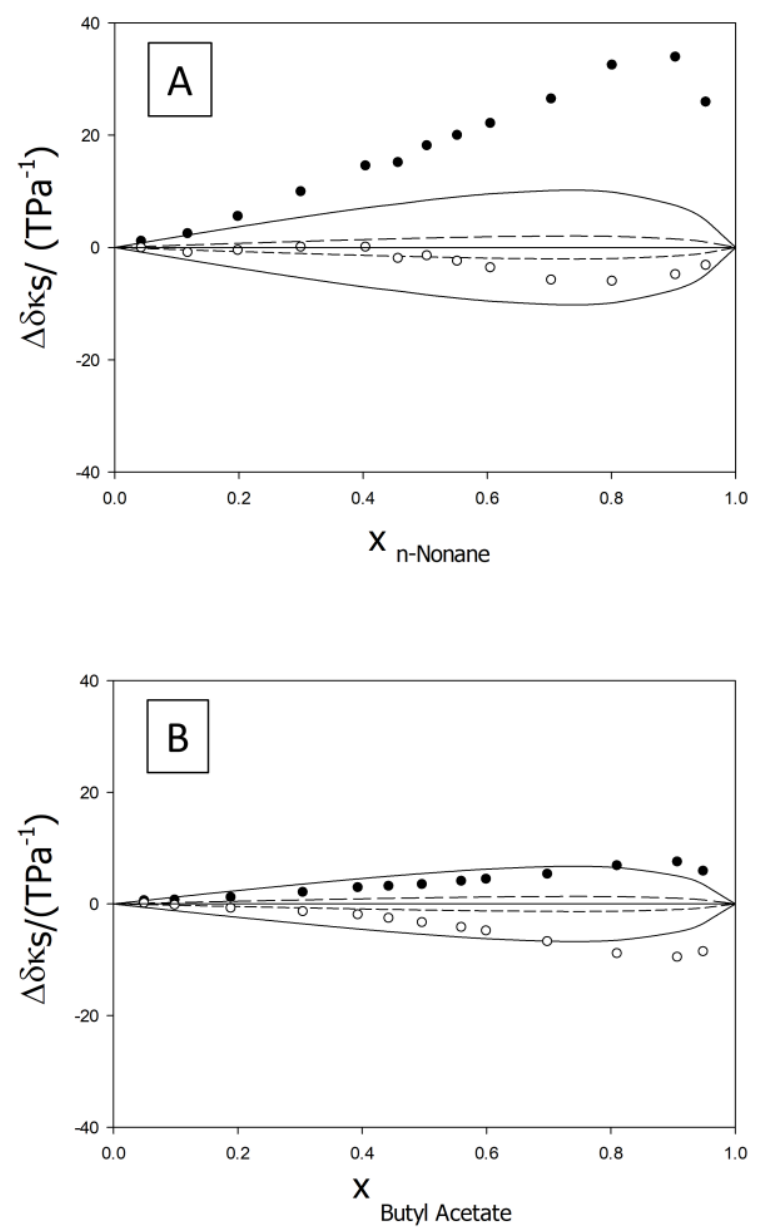

Fig.5. Comparison of experimental and estimated data by Junjie (•) and Nomoto (०) for change of isentropic compressibilities at $298.15 \mathrm{~K}$, for ((a) n-nonane and (b) butyl acetate) + olive oil mixtures. The lines (----, $1 \%$ deviation; - $5 \%$ deviation) indicate percentual deviation from experimental values as a reference.

As above commented upon, ester solvents introduce a relative polar charge around glicerides. Vinyl acetate is the most expansive case due to a combination of low molar mass and polar ester functional group - unsaturated group. This structure avoids an easy bulk packing among long chain triglycerides. On the other hand, isopropyl acetate gathers the highest contractive behavior into olive oil mixtures. This solvent shows an unique polar group besides a flat structure of apolar nature. As observed this solvent shows small negative values of change of isentropic compressibility, as corresponding with a potential low contractive solvent if compared with $n$ alkane tendency [16]. In what is referred to theoretical estimation, mixing rules for refractive index were applied
[27], as well as different models for ultrasonic velocity [28-33] were applied. The best results in terms of estimation were gathered into Figures 4 and 5 for both derived properties, respectively. For the n-alkanes + olive oil mixtures, the Arago-Biot, Dale-Gladstone, Newton and Oster equations (eqs. 6, 8, 9, 10) show a better accuracy than the other studied procedures. For example, in Figure 4A and 4B experimental data of the authors for n-nonane + olive oil and butyl acetate + olive oil, respectively, are compared with the values estimated by the best methods. A good concordance are shown between our experimental data and those estimated, which gather a difference better than $1 \%$ for GladstoneDale equation and better than $5 \%$ for Newton and Oster equations for n-nonane mixtures. These methods gather better estimation capability for ester mixtures, all of them reaching deviations better than 1\% (Figure 4B) when butyl acetate mixtures are analysed. For the estimation of ultrasonic velocities of the mixtures, two empirical models: Junjie and Nomoto were used for graphic presentation (Figures 5A-5B). These ultrasonic velocity estimation methods achieve results around $5 \%$ in deviation for n-nonane and butyl acetate. Only Junjie method shows greater deviations at higher hydrocarbon compositions as could be observed into Figure 5A. Attending to the obtained results, it could be concluded that the applied methods allow, at least, gathering a qualitative estimation of the mixing trend in terms of optical or acoustical properties at the studied range of temperatures for this kind of mixtures.

\section{Acknowledgments}

The authors would like to acknowledge National Council for Scientific and Technological Development (M. Iglesias - Chamada CNPq $\mathrm{N}^{\circ}$ 29/2019, Processo 313601/2019-4 and R. S. Andrade - Chamada MCTIC/CNPq No 28/2018, Processo 438376/2018-8) for its support in developing this research.

\section{Nomenclature}

$\mathrm{B}_{\mathrm{i}}$ - characteristic molecular volume $\left(\mathrm{m}^{3}\right)$

$\mathrm{B}_{\mathrm{ij}}-$ Fitting parameters of eq. 4

$\mathrm{K}$ - mathematical function of temperature

$\mathrm{L}_{\text {mix }}$ - intermolecular free length of a mixture $(\mathrm{cm})$

$\mathrm{M}_{\mathrm{eff}}$ - effective molar mass of a mixture $\left(\mathrm{gmol}^{-1}\right)$

$\mathrm{M}_{\mathrm{i}}$ - molar mass of a pure compound $\left(\mathrm{gmol}^{-1}\right)$

$\mathrm{M}_{\mathrm{oil}}$ - molar mass of an oil $\left(\mathrm{gmol}^{-1}\right)$

$\mathrm{M}_{\mathrm{i}}$ - molar mass of each fatty acid according to the concentration analysis $\left(\mathrm{gmol}^{-1}\right)$

$\mathrm{M}_{\mathrm{CH}-\mathrm{C}-\mathrm{CH}}$ - molar mass contributions of the triglyceride molecule axis fraction without three protons $\left(\mathrm{gmol}^{-1}\right)$

M degree of the polynomial expansion (eq. 3)

NFA - number of fatty acid found by chromatographic analysis

$\mathrm{n}_{\mathrm{D}}$ - refraction index of a mixture

$\mathrm{n}_{\mathrm{Di}}-$ refraction index of a pure component

$\mathrm{n}_{\mathrm{DAT}}-$ number of experimental data (eq. 4 )

$\mathrm{N}$ - number of components into a mixture

$\mathrm{P}_{\text {mix }}$ - mixing thermodynamic magnitude

$\mathrm{P}_{\mathrm{i}}$ - thermodynamic magnitude of a pure compound

$\mathrm{R}_{\mathrm{i}}-$ molar sound velocity of a pure compound $\left(\mathrm{cm}^{3} \mathrm{~mol}^{-}\right.$ $\left.{ }^{1}\left(\mathrm{~ms}^{-1}\right)^{1 / 3}\right)$

$\mathrm{S}$ degree of the polynomial expansion (eq. 3)

$\mathrm{S}$ - collision factor

(eq. 18) 
$\mathrm{T}$ - temperature $(\mathrm{K})$

$\mathrm{u}-$ ultrasonic velocity of a mixture $\left(\mathrm{ms}^{-1}\right)$

$\mathrm{u}_{\mathrm{i}}-$ ultrasonic velocity of a pure compound $\left(\mathrm{ms}^{-1}\right)$

$\mathrm{u}_{\infty}$ - ultrasonic velocity constant $\left(1600 \mathrm{~ms}^{-1}\right)$

$\mathrm{V}_{\mathrm{i}}$ - molar volume of a pure compound $\left(\mathrm{cm}^{3} \mathrm{~mol}^{-1}\right)$

$\mathrm{V}_{\text {mix }}-$ molar volume of a mixture $\left(\mathrm{cm}^{3} \mathrm{~mol}^{-1}\right)$

$\mathrm{x}_{\mathrm{i}}-$ molar fraction of $\mathrm{i}$ compound

$\mathrm{x}_{\mathrm{j}}$ - molar fraction of $\mathrm{j}$ compound

$\mathrm{Y}_{\mathrm{i}}$ - molar surface area of a pure compound $\left(\mathrm{cm}^{2}\right)$

$\mathrm{Z}$ - value of a property (eq. 4)

$\mathrm{Z}_{\mathrm{i}}$ - specific acoustic impedance of a pure compound $\left(\mathrm{gcm}^{-3} \mathrm{~ms}^{-1}\right)$

$\delta \mathrm{P}$ - change of a thermodynamic magnitude $\mathrm{P}$ for a mixture

$\delta \mathrm{P}_{\mathrm{ij}}$ - change of a thermodynamic magnitude $\mathrm{P}$ for $\mathrm{a}$ mixture of $i$ and $j$ compounds

$\phi$ - volume fraction

$\rho_{\text {mix }}$ - density of a mixture $\left(\mathrm{gcm}^{-3}\right)$

$\rho_{\mathrm{i}}$ - density of a pure component $\left(\mathrm{gcm}^{-3}\right)$

$\sigma-$ root mean square deviation (eq. 4)

\section{References:}

[1] S. Manjula, R. Subramanian, "Membrane technology in degumming, dewaxing, deacidifying, and decolorizing edible oils," Crit. Rev. Food Sci. Nutr., 46(7), 569-592, 2006.

[2] X. Hu, Q. Li, G. Xu, Z. Gao, "Study on technical process of deacidifying and dewaxing rice bran oil through ethanol extraction," J. Chin. Cer. and Oils Assoc., 28(8), 956-962, 2013.

[3] S. Ariponnammal, "A novel method of using refractive index as a tool for finding the adultration of oils," Res. J. of Recent Sci., 1(7), 77-79, 2012.

[4] Eugene Hecht., Optics, Addison-Wesley (2002)

[5] S. S. Arya, S. Ramanujam, P. K. Vijayaraghavan, "Refractive index as an objective method for evaluation of rancidity in edible oils and fats," J. Am. Oil Chem.' Soc., 46 (1), 28-30, 1969.

[6] S. K. Mahammad Ali, B. Ali, "Velocity of ultrasound in commonly used vegetable oils at low frequencies," Int. J. Sci. Env. Tech., 3(5), 1803-1809, 2014.

[7] G. V. Rama Rao, A. Viswanatha Sarma, J. Siva Rama Krishna, C. Rambabu, "Theoretical evaluation of ultrasonic velocities in binary liquid mixtures of ochlorophenol at different temperatures," Indian J. Pure Ap. Phy., 43(5), 345-354, 2005.

[8] P. Vasantharani, S. Muthu Shailaja, A. N. Kannappan, R. Ezhil Pavai, "Theoretical evaluation of ultrasonic velocity in organic liquid mixtures," J. Appl. Sci., 8(12), 2329-2332, 2008.

[9] J. D. Pandey, A. K. Singh, R. Dey, "Novel approach for prediction of ultrasonic velocity in quaternary liquid mixtures," Pramana, 64(1), 135-139, 2005.
[10] J. D. Pandey, R. Dey, D. K. Dwivedi, "Ultrasonic velocity of binary systems at elevated pressures," Pramana, 52(2), 187-193, 1999.

[11] T. Sumathi, J. U. Maheswari, "Ultrasonic and theoretical studies of some ternary liquid mixtures at Various temperatures," Indian J. Pure Ap. Phy., 47(11), 782-786, 2009.

[12] S. Baluja, S. Oza, "Studies of some acoustical properties in binary solutions," The Journal of Pure and Applied Ultrasonics, 24, 580-583, 2002.

[13] A. Ali, A. Yasmin, A. K. Nain, "Study of intermolecular interactions in binary liquid mixtures through ultrasonic speed measurement," Indian J. Pure Ap. Phy., 40(5), 315-322, 2002.

[14] M. Rastogi, A. Awasthi, M. Gupta, J. P. Shukla, "Ultrasonic investigations of X...HO bond complexes," Indian J. Pure Ap. Phy., 40(4), 256-263, 2002.

[15] A. Ali, A. K. Nain, S. Hyder, "Ultrasonic study of molecular interaction in binary liquid mixtures at 308.15 K,”J. Pure App. Ultrasonics, 23, 73-79, 2001.

[16] C. Gonzalez, J.M. Resa, J. Lanz, "Excess volumes of binary mixtures that contain olive oil with alkyl and vinyl acetates," J. Am. Oil Chem. 'Soc., 77(9), 985990, 2000.

[17] C. Gonzalez, J.M. Resa, J. Lanz, M. Iglesias, J.M. Goenaga, "Measurements of density and refractive index of soybean oil + short aliphatic alcohols," Int. J. Thermophys., 27(5), 1463-1481, 2006.

[18] C. Gonzalez, J.M. Resa, R.G. Concha, J.M. Goenaga, "Enthalpies of mixing and heat capacities of mixtures containing acetates and ketones with corn oil at 25 ${ }^{\circ}$ C," J. Food Eng., 79(3), 1104-1109, 2007.

[19] F. Tanajura, R.S. Andrade, M. Iglesias, C. Gonzalez, "Thermodynamic properties of peanut, canola and rosa mosqueta oil," Elixir Int. J., 101, 43587-43592, 2016.

[20] R.S. Andrade, G.A. Ferreira, D. Camargo, M. Iglesias, "Thermodynamic properties of palm oil (Elaeis guineensis) and evening primrose seed oil (Oenothera biennis) as a function of temperature," World Wide Journal of Multidisciplinary Research and Development, 2, 38-43, 2016.

[21] J. Riddick, W. Bunger, T.K. Sakano, Organic Solvents. Wiley Interscience, New York, 1986.

[22] J.G. Baragi, M.I. Aralaguppi, M.Y. Kariduraganavar, S.S. Kulkarni, A.S., Kittur, T.M. Aminabhavi, "Excess properties of the binary mixtures of methylcyclohexane + alkanes $\left(\mathrm{C}_{6}\right.$ to $\left.\mathrm{C}_{12}\right)$ at $\mathrm{T}=$ 298.15 K to T $=308.15$ K," J. Chem. Thermodyn., 38, 75-83, 2006. 
[23] L. Sarkar, M.N. Roy, "Studies on liquid-liquid interactions of some ternary mixtures by density, viscosity, ultrasonic speed and refractive index measurements," Thermochim. Acta, 496(1-2), 124$128,2009$.

[24] J. de D. Alvarado, "Propiedades mecánicas de aceites y grasas," Grasas y aceites, 46(4-5), 264-269, 1995.

[25] J.N. Coupland, D.J. McClements, "Physical properties of liquid edible oils,". J. Am. Oil Chem. Soc., 74(12), 1559-1564, 1997.

[26] L.C. Lynnworth, "Industrial applications of ultrasound - a review. II. Measurements, tests and process control using low intensity ultrasound," \{IEEE\} Trans. Sonics Ultrason., 54(22), 71-101, 1975.

[27] M. Iglesias, B. Orge, J. Tojo, "Refractive indices and densities of the ternary mixtures acetone + methanol + water and acetone + methanol + 1-butanol at 298.15 K," Fluid Phase Equilib., 126(2), 203-223, 1996.

[28] F. Danusso, "Ultrasonic velocity and adiabatic compressibility of liquid mixtures,". Atti Accad. Naz. Lincei, 10, 235-242, 1951.

[29] O. Nomoto, "Sound velocity in liquids in relation to cell model theory," J. Phys. Soc. Jpn., 18(10), 15261528,1963 .

[30] V. Rajendran, A.N. Kannappan, "Excess isentropic compressibility and computation of sound velocity in ternary liquid mixtures,". Indian J. Phys., 68, 131-138, 1994.

[31] M. Kalidoss, R. Srinivasamoorthy, R., "Ultrasonic study of ternary liquid mixtures of cyclohexanone + 1,2 dicholorotane + n-propanol, + n-butanol," J. Pure Applied Ultrason., 19(1), 9-15, 1997.

[32] W. Schaffs, Molekulara Bustik, Berlin: SpringerVerlag, Chapters XI and XII, 1963.

[33] B. Jacobson, "Ultrasonic velocity in liquids and liquid mixtures," J. Chem. Phys., 20(5), 927-928, 1952.

[34] C. González, M. Iglesias, J. Lanz, J. M. Resa, "Temperature dependence of excess molar volumes in (n-alkanes $\left(\mathrm{C}_{6}-\mathrm{C}_{9}\right)$ or alcohol $\left.\left(\mathrm{C}_{2}-\mathrm{C}_{4}\right)\right)+$ olive oil mixtures," Thermochim. Acta, 328, 277-296, 1999. 